\title{
Multivariate dependence analysis via tree copula models: An application to one-year forward energy contracts
}

\author{
Federico Bassetti a , Maria Elena De Giuli b , Enrica Nicolino b, c, Claudia Tarantola b, * \\ a Department of Mathematics, University of Pavia, Via Ferrata 5, Pavia, 27100, Italy \\ ${ }^{b}$ Department of Economics and Management, University of Pavia, Via San Felice 5, Pavia, 27100, Italy \\ ${ }^{c}$ RGI S.p.A., Corso Svizzera 185, Torino, 10149, Italy
}

\begin{abstract}
We propose a novel multivariate approach for dependence analysis in the energy market. The method- ology is based on tree copulas and GARCH type processes. We use it to study the dependence structure among the main factors affecting energy price, and to perform portfolio risk evaluation. The temporal dynamic of the examined variables is described via a set of GARCH type models where the joint distribution of the standardised residuals is represented via suitable tree copula structures. Working in a Bayesian framework, we perform both qualitative and quantitative learning. Posterior summaries of the quantities of interest are obtained via MCMC methods.
\end{abstract}

\section{Introduction}

In recent years, the behaviour of the energy market has assumed a crucial role in the global economy, impacting and influencing both economic and social activities. Energy price directly affects industrial costs, becoming a fundamental element in the decision-making process of companies and entrepreneurs. The energy price is related to the cost and quantity of raw materials used to produce it. Moreover, since 2005, it is also related to the price for carbon emission $\left(\mathrm{CO}_{2}\right)$. Indeed, when applying the Kyoto Protocol, the European Union Emissions Trading Scheme (EU-ETS) of 2005 set up caps for the $\mathrm{CO}_{2}$ emissions of plants. Installations can increase emissions above their caps by acquiring emission allowances. Furthermore, installations with emissions below caps are allowed to sell unused allowances. Permits can be traded in spot, future and option markets and the power sector is a key player in the EU-ETS, see e.g. Reinaud (2007). Finally, the elements determining the energy price have become increasingly interconnected in the last years.

In this paper, we use Bayesian AR-GARCH copula models to study the behaviour and the connections among the main factors affecting energy price (coal, gas, oil and $\mathrm{CO}_{2}$ prices). Our aim is to identify the dependence structure characterising the market, with

\footnotetext{
* Corresponding author.

E-mail addresses: federico.bassetti@unipv.it (F. Bassetti), elena.degiuli@unipv.it

(M.E. De Giuli), enrica.nicolino@rgigroup.com (E. Nicolino),

claudia.tarantola@unipv.it (C. Tarantola).
}

particular attention to tail behavior. We focus on two representative European markets, the Italian and the German. For both markets we consider daily observations of one year forward contracts subscribed in 2014. Differently from the German case, only in 2014 Italian power prices were traded on a regulated market. In order to investigate the effect of this event we also analyse the Italian market in the period 2013-2016. We work in terms of monthly logarithmic return rates and we model their temporal dynamic via AR-GARCH processes. We account for the dependence between the variables by fitting alternative copula models to the distribution of the standardised residuals.

We perform both qualitative and quantitative Bayesian analysis, and we show how suitable pictorial representations of the dependence structure of the processes can be obtained. Finally, we illustrate how market risk of energy portfolios can be easily estimated via Bayesian predictive measures.

The estimated dependence structures are in line with some specific characteristics of the current energy market. In particular, we observe that the price of Brent (one of the major classifications of oil) has a marginal influence on the power price and the commodities that mostly impact the energy price are natural gas and coal. Furthermore, for the Italian case we find that the pairwise dependence between variables increases for almost all the examined quantities from 2013 to 2016.

Among possible alternative models for dependence analysis, we focus on copula functions, which are nowadays very popular in finance, insurance, econometrics and recently in the analysis of commodity markets; see e.g. Aas, Czado, Frigessi, and Bakken 
(2009), Czado, Gärtner, and Min (2011), Arreola Hernandez (2014), Dalla Valle, De Giuli, Tarantola, and Manelli (2016), Jaschke (2014), Laih (2014), Marimoutou and Soury (2015), Wen, Wei, and Huang (2012), Wu, Chung, and Chang (2012) and Oh and Patton (2017). Although there are different types of bivariate copulas available, the choice of multivariate copulas is rather limited, due to computational and theoretical limitations. To overcome this issue, Joe (1996) introduced the Pair Copula Construction (PCC), as an instrument for building flexible multivariate copulas starting from a set of bivariate ones, referred to as linking copulas. The core of this approach is its graphical representation, called R-vines, that consists of a nested set of trees, each edge of which is associated with a linking copula, see Bedford and Cooke (2001, 2002). Unfortunately, $\mathrm{R}$-vines present a combinatorial complexity that may create difficulties in structural learning and parameter estimation both in frequentist and in Bayesian settings. In the frequentist approach, a two steps estimation procedure, known as Inference Function for Margins (IFM) proposed by Joe (1997), is usually applied. Also in the Bayesian framework it is common the use of a suitable two steps procedure where the underlying copula structure is selected a priori, see e.g. Hofmann and Czado (2010), Min and Czado (2010) and Czado, Gärtner, and Min (2011) . Recently, Gruber and Czado $(2014,2015)$ developed a Bayesian approach for vine with structural learning. Nevertheless, due to the nested structure of the $\mathrm{R}$-vine, the algorithms used to simulate from the posterior distributions are computationally demanding.

In order to reduce the complexity of the learning procedure and develop an efficient Bayesian approach to jointly estimate the copula structure and its parameters, in this paper we rely on tree copula models introduced by Kirshner (2007). Tree copulas are truncated Rvines, see Kurowicka (2011), whose underlying graphical structure, simpler than the R-vine structure, allows the inference procedure to be simplified. Furthermore, they provide a pictorial representation of the dependence structure that is easy to explain to non-experts. Nevertheless, considering only tree structures may be too restrictive to represent a realistic dependence among variables. Hence, following Silva and Gramcy (2009) and Elidan (2013), we also examine finite and infinite mixture of tree copulas. In the latter case we assume a non-parametric Dirichlet Process prior.

The use of Bayesian techniques in contrast to frequentist methods is motivated by the fact that the latter are not asymptotically efficient when applied to copula models, see Joe (2005). Moreover, in the Bayesian setting parameters uncertainty can be considered in the prediction. Another advantage of our approach based on Markov Chain Monte Carlo (MCMC) methods is that it allows mixture models to be estimated easily. Finally, portfolio predictive cumulative distributions, risk measures and credible intervals for all the estimated parameters can be straight forwardly approximated by using the output of the MCMC.

The outline of the paper is as follows. In Section 2 our tree copula models are presented. In Section 3 the Bayesian estimation methodology is outlined. Section 4 describes the application of the proposed methodology to the analysis of real data. Readers primarily interested in the application may wish to browse lightly through Sections 2-3 and focus on Section 4. Concluding remarks are given in Section 5. The details of the MCMC algorithms and fur- ther results on simulated data are provided in the Supplementary Material.

\section{AR-GARCH copula models specification}

In order to describe the dynamic of the prices of the commodities we rely on AR-GARCH copula models. Let $S_{t, k}$ be the price at

day $t$ of commodity $k$, and $X_{t, k}=\log \left\{S_{t+20, k} / S_{t, k}\right\}$ be the corresponding monthly logarithmic return rate. Varying $t$ over the set of working days, we obtain for each commodity $k=1, \ldots, N$ a time series $\left(X_{k, t}\right)$ that we model via an $A R(p)-\operatorname{GARCH}(q, r)$ structure. More precisely,

$$
\begin{aligned}
X_{k, t} & =\sum_{i=1}^{p} a_{k, i} X_{k, t-i}+\varepsilon_{k, t}, \\
\varepsilon_{k, t} & =\sigma_{k, t} Z_{k, t}, \\
\sigma_{k, t}^{2} & =\sigma_{k}^{2}+\sum_{i=1}^{q} b_{k, i} \sigma_{k, t-i}^{2}+\sum_{j=1}^{r} c_{k, j} \varepsilon_{k, t-j}^{2} .
\end{aligned}
$$

Setting $q$ and $r$ equal to 0 , one obtains an $A R(p)$ model with $\sigma_{k, t}^{2}=$ $\sigma_{k}^{2}$ for every $t \geq 1$.

The vectors $Z_{\boldsymbol{t}}=\left(Z_{1, t}, \ldots, Z_{N, t}\right)$ for $t=1, \ldots, T$ are usually assumed to be independent and identically distributed. A common assumption is that $Z_{k, t}=\varepsilon_{k, t} / \sigma_{k, t}$ are standardised residuals normally distributed with zero mean and unit variance, and are jointly normally distributed with unknown correlation matrix. As an alternative, in this work we propose copula based models for the vector $\boldsymbol{Z}_{\boldsymbol{t}}$ of the standardised residuals, see Section 2.4.

In Sections 2.1-2.3 we briefly introduce copula functions, the related notation and terminology needed to define our models.

\subsection{Copula functions}

A popular and efficient tool in multivariate dependence analysis is the copula function. The advantage of copulas is the ability to obtain the joint multivariate distribution embedding the dependence structure of the variables. A copula is a multivariate distribution with uniform margins on the unit interval. It is used to couple one-dimensional marginal distributions in order to obtain the corresponding joint multivariate distribution. Sklar's theorem (Sklar, 1959) states that any $\mathrm{N}$-dimensional cumulative distribution function (cdf) $F$, with univariate cumulative marginal distributions $F_{1}, \ldots, F_{N}$, can be written as $F\left(z_{1}, \ldots, z_{N}\right)=C\left(F_{1}\left(z_{1}\right), \ldots, F_{N}\left(z_{N}\right)\right)$, where $C$ is a suitable copula function. Consequently, if $F$ is absolutely continuous, the corresponding joint probability density function (pdf) is given by

$$
f\left(z_{1}, \ldots, z_{N}\right)=c\left(F_{1}\left(z_{1}\right), \ldots, F_{N}\left(z_{N}\right)\right) f_{1}\left(z_{1}\right) \ldots f_{N}\left(z_{N}\right),
$$

where $c$ is the copula density function.

\subsection{Tree copula}

As mentioned in Section 1 graphical models can be used to simplify the construction of multivariate copulas. In a graphical model, the structure of the graph provides a pictorial representation of the conditional independence relationships between the variables; for a detailed presentation and the relevant terminology see Lauritzen (1996).

In this paper, we consider a Markov tree model, a particular type of graphical model having as underling graph an undirected tree with set of nodes $\mathcal{V}=\{1, \ldots, N\}$ and set of edges $\mathcal{E}$ (unordered pair of nodes). A random variable is associated with each node of the tree and the global Markov property is used to read conditional independencies among them. According to this property, disconnected sets of variables are conditionally independent given a separating set. Since a tree is uniquely defined by its edge set, in the following we use $\mathcal{E}$ to denote the tree structure. We indicate with $\mathscr{E}_{N}$ the set, of cardinality $N^{N-2}$, of all tree structures with $N$ nodes.

If $\boldsymbol{Z}$ is a random vector with multivariate (positive) pdf $f$ on $\mathcal{Z} \subset$ $\mathbb{R}^{N}$ represented by a Markov tree $\mathcal{E}$, then its joint density can be factorised as

$f\left(z_{1}, \ldots, z_{N}\right)=\left[\prod_{(l, m) \in \mathcal{E}} \frac{f_{l, m}\left(z_{l}, z_{m}\right)}{f_{l}\left(z_{l}\right) f_{m}\left(z_{m}\right)}\right] \prod_{i=1}^{N} f_{i}\left(z_{i}\right)$, 


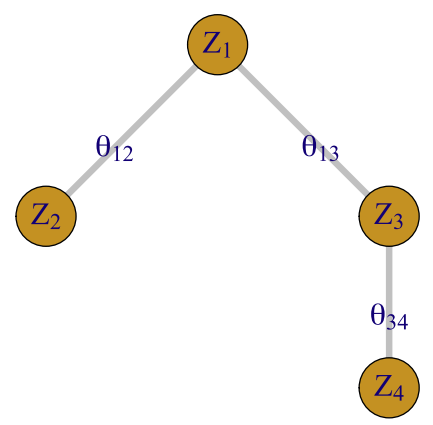

Fig. 1. Graphical representation of a tree copula on 4 variables.

where $f_{l}$ is the marginal density of $Z_{l}$ and $f_{l, m}$ is the joint density of $\left(Z_{l}, Z_{m}\right)$

Following the tree copula construction of Kirshner (2007), we represent each density $f_{l, m}$ in (2) via the corresponding bivariate linking copula density $c_{l, m}$ associated with the edge $(l, m)$. Hence, Eq. (2) can be re-written as

$f\left(z_{1}, \ldots, z_{N}\right)=\prod_{(l, m) \in \mathcal{E}} c_{l, m}\left(F_{l}\left(z_{l}\right), F_{m}\left(z_{m}\right)\right) \prod_{i=1}^{N} f_{i}\left(z_{i}\right)$,

where $F_{l}$ and $F_{m}$ are the marginal cdfs of $Z_{l}$ and $Z_{m}$.

Conversely, given a tree structure $\mathcal{E}$ and a family of a bivariate copula $c_{l, m}\left(u_{l}, u_{m} \mid \theta_{l, m}\right)$ (parameterised through a parameter $\left.\theta_{l, m}\right), \quad c_{\theta}\left(u_{1}, \ldots, u_{N}\right)=\prod_{(l, m) \in \mathcal{E}} c_{l, m}\left(u_{l}, u_{m} \mid \theta_{l, m}\right)$ is an admissible copula density. To simplify the notation, if $m$ is the parent node of $l$ in the directed version of $\mathcal{E}$ with root node 1 , we set $c_{\theta_{l, m}}\left(u_{l}, u_{m}\right)=c_{l, m}\left(u_{l}, u_{m} \mid \theta_{l, m}\right)$; otherwise if $l$ is the parent node of $m, c_{\theta_{m, l}}\left(u_{m}, u_{l}\right)=c_{l, m}\left(u_{l}, u_{m} \mid \theta_{l, m}\right)$. Consequently, $f_{\theta}\left(z_{1}, \ldots, z_{N}\right)=\prod_{(l, m) \in \mathcal{E}} c_{\theta_{l, m}}\left(F_{l}\left(z_{l}\right), F_{m}\left(z_{m}\right)\right) \prod_{i=1}^{N} f_{i}\left(z_{i}\right)$, is a density with margins $f_{i}, i=1, \ldots, N$.

For example, for the tree copula reported in Fig. 1, the joint density factorises as

$$
\begin{aligned}
f\left(z_{1}, z_{2}, z_{3}, z_{4}\right)= & c_{\theta_{1,2}}\left(F_{1}\left(z_{1}\right), F_{2}\left(z_{2}\right)\right) c_{\theta_{1,3}}\left(F_{1}\left(z_{1}\right), F_{3}\left(z_{3}\right)\right) c_{\theta_{3,4}} \\
& \times\left(F_{3}\left(z_{3}\right), F_{4}\left(z_{4}\right)\right) \prod_{k=1}^{4} f_{k}\left(z_{k}\right) .
\end{aligned}
$$

\subsection{Mixture of tree copula}

In order to overcome the restrictions imposed by the tree structure, we consider copula functions obtained as the mixture of tree copulas. This strategy allows us to preserve the relative low complexity of the Markov tree structures, taking into account richer dependencies between the variables. A mixture of Markov tree copulas is given by

$c\left(u_{1}, \ldots, u_{N}\right)=\sum_{d=1}^{D} w_{d} \prod_{(l, m) \in \mathcal{E}_{d}} c_{\theta_{l, m}^{(d)}}\left(u_{l}, u_{m}\right)$,

where $D \leq+\infty$ is the number of mixture components, $\left(w_{d}\right)_{d=1, \ldots, D}$ are positive weights with $\sum_{d=1}^{D} w_{d}=1, \mathcal{E}_{d}(d=1, \ldots, D)$ is the tree structure of the $d$ th component of the mixture, and $\left\{\theta_{l, m}^{(d)}\right\}$ are the copula parameters corresponding to the tree structure $\mathcal{E}_{d}$.

A possible drawback of the mixture of tree copula is that its graphical model cannot be straightforwardly identified. See Meilă and Jordan (2000) for more details.

\subsection{Distribution of the standardised residuals}

In our AR-GARCH models we consider two alternative copulabased distributions for the vectors of standardised residuals.
According to the first one, we assume that for any fixed $t$ the dependence structure among the standardised residuals is given by a tree copula distribution with unknown underlying structure $\mathcal{E}$. Therefore, the pdf of the vector $\boldsymbol{Z}_{t}$ is given by

$f_{\boldsymbol{Z}_{t}}\left(z_{1, t}, \ldots, z_{N, t} \mid \boldsymbol{\theta}, \mathcal{E}\right)=\prod_{(l, m) \in \mathcal{E}} c_{\theta_{l, m}}\left(F_{v_{l}}\left(z_{l, t}\right), F_{v_{m}}\left(z_{m, t}\right)\right) \prod_{k=1}^{N} f_{v_{k}}\left(z_{k, t}\right)$,

where $\boldsymbol{\theta}$ is the collection of all the copula parameters $\theta_{l, m}$ with $(l, m) \in \mathcal{E}, F_{v_{k}}$ is the marginal cumulative distribution function with parameter $v_{k}$ and $f_{v_{k}}$ the corresponding density. In the second model, we represent the joint density of the standardised residuals via a mixture of tree copulas, i.e.

$$
\begin{aligned}
f_{Z_{t}}\left(z_{1, t}, \ldots, z_{N, t} \mid \boldsymbol{w}, \mathfrak{E}, \Theta\right) \\
\quad=\sum_{d=1}^{D} w_{d} \prod_{(l, m) \in \mathcal{E}_{d}} c_{\theta_{l, m}^{(d)}}\left(F_{v_{l}}\left(z_{l, t}\right), F_{v_{m}}\left(z_{m, t}\right)\right) \prod_{k=1}^{N} f_{v_{k}}\left(z_{k, t}\right),
\end{aligned}
$$

where $\boldsymbol{w}=\left(w_{1}, \ldots, w_{D}\right), \quad \mathcal{E}=\left\{\mathcal{E}_{1}, \ldots, \mathcal{E}_{D}\right\}, \quad \Theta=\left\{\boldsymbol{\theta}_{1}, \ldots, \boldsymbol{\theta}_{D}\right\}, \quad \boldsymbol{\theta}_{d}$ denoting the collection of the copula parameters $\theta_{l, m}^{(d)}$ for the $d$ th component.

The assumption of normality for the marginal distribution function $F_{v_{k}}$ of the standardised residuals may be not adequate due to possible heavy tails and asymmetry in the data. Hence, in addition to the case of Normal residuals, we also consider the case in which the standardised residuals follow a Skew Student- $t$ distribution of parameters $v_{k}=\left(\lambda_{k}, \eta_{k}\right)$.

Following Hansen (1994), the Skew- $t$ density with parameters $\lambda$ (skewness) and $\eta$ (degree of freedom) is defined as

$f_{\lambda, \eta}(z)= \begin{cases}b c\left(1+\frac{1}{\eta-2}\left(\frac{b z+a}{1-\lambda}\right)^{2}\right)^{-(\eta+1) / 2} & z<-a / b \\ b c\left(1+\frac{1}{\eta-2}\left(\frac{b z+a}{1+\lambda}\right)^{2}\right)^{-(\eta+1) / 2} & z \geq-a / b\end{cases}$

with $\eta>2$ and $-1<\lambda<1$. The constants $a, b$ and $c$ are given by $a=4 \lambda c\left(\frac{\eta-2}{\eta-1}\right), \quad b^{2}=1+3 \lambda^{2}-a^{2}, \quad$ and $\quad c=$ $\Gamma\left(\frac{\eta+1}{2}\right) / \sqrt{\pi(\eta-2) \Gamma(\eta / 2)}$.

Hansen (1994) shows that this is a proper density function with mean 0 and unit variance. Furthermore, if $\lambda=0$ the Skew- $t$ is reduced to the standard Student- $t$ distribution. If $\lambda>0(\lambda<0)$, then this function is positively (negatively) skewed.

\subsection{Linking copulas}

We assume that every bivariate copula density $c_{\theta_{l, m}}$ belongs to a specific family depending on a parameter $\theta_{l, m}$. We focus on two well-known family of copulas for tail dependence, Gumbel and Clayton, and their rotations (Double Gumbel and Double Clayton). These copulas have been widely used in applied analyses to study tail dependence between variables of interest. More sophisticated families of copulas for tail dependence can be effectively approximated for practical purposes by Gumbel and Clayton ones, see Demarta and McNeil (2005).

Double Gumbel and Double Clayton copulas are based on the rotations of standard Gumbel and Clayton copula family. The Gumbel copula is given by $C^{G}(u, v)=$ $\exp \left\{-\left[(-\log u)^{\phi}+(-\log v)^{\phi}\right]^{1 / \phi}\right\}$, where $\phi$ is a parameter assuming value in $[1, \infty)$. The Clayton copula is defined as $C^{C}(u, v)=\left[\max \left(u^{-\phi}+v^{-\phi}-1,0\right)\right]^{-1 / \phi}$, where $\phi \in(0, \infty)$. In order to define Double Clayton and Double Gumbel copulas, we first reparameterise the copulas defined above in term of Kendall's tau measure; $\tau=(\phi-1) / \phi$ for the Gumbel copula, while $\tau=\phi /(2+\phi)$ in the case of Clayton copula. 
Subsequently, rotations are used to obtain the Double Clayton and Double Gumbel copulas. If $c^{G}(u, v ; \tau)$ is the Gumbel copula density reparameterised by the Kendall's tau, the Double Gumbel copula of first kind of parameter $\tau$ is defined by

$c^{D G_{1}}(u, v ; \tau)= \begin{cases}c^{G}(u, v ; \tau) & \text { for } \tau>0 \\ c^{G}(1-u, v ;-\tau) & \text { for } \tau<0,\end{cases}$

while the Double Gumbel copula of second kind is

$c^{D G_{2}}(u, v ; \tau)= \begin{cases}c^{G}(1-u, 1-v ; \tau) & \text { for } \tau>0 \\ c^{G}(u, 1-v ;-\tau) & \text { for } \tau<0 .\end{cases}$

In an analogous way we define the Double Clayton of first and second kind, $\mathrm{DC}_{1}$ and $\mathrm{DC}_{2}$, in term of rotations and Kendall's tau. Therefore, any linking copula $c_{\theta_{l, m}}$ is completely described by the parameter $\theta_{l, m}=\left(\tau_{l, m}, \zeta_{l, m}\right)$ taking values in $(-1,1) \times \mathcal{H}$, where $\mathcal{H}=\left\{D G_{1}, D G_{2}, D C_{1}, D C_{2}\right\}$.

\section{Methodology}

In this section, we illustrate how to perform Bayesian inference for AR-GARCH copula models. We denote with $\Phi$ the collection of all the parameters describing the copula structures (for instance in the case of the simple tree copula distribution one has $\Phi=\{\boldsymbol{\theta}, \mathcal{E}\})$, with $\boldsymbol{v}=\left(v_{1}, \ldots, v_{N}\right)$ the collection of the parameters of the marginal distributions and with $\boldsymbol{A}$ the collection of the parameters of the marginal time series models. In a fully Bayesian approach, if $\pi(\cdot)$ is the prior density on the parameters $(\Phi, \boldsymbol{v}, \boldsymbol{A})$ and $L\left(\mathcal{O}_{T} \mid \Phi, \boldsymbol{v}, \boldsymbol{A}\right)$ is the likelihood of the observations $\mathcal{O}_{T}=\left\{\left(x_{1, t}, \ldots, x_{N, t}\right), t=1, \ldots, T\right\}$, we obtain the posterior density using Bayes' theorem as

$\pi\left(\Phi, \boldsymbol{v}, \boldsymbol{A} \mid \mathcal{O}_{T}\right) \propto L\left(\mathcal{O}_{T} \mid \Phi, \boldsymbol{v}, \boldsymbol{A}\right) \pi(\Phi, \boldsymbol{v}, \boldsymbol{A})$.

In principle, from the posterior distribution one can extract all the information needed for inference. In particular, one can compute posterior quantities, such as the mean or mode, as estimators for the parameters and evaluate predictive distributions for forecasting. Often the posterior distribution does not have a closed-form analytical expression. One possibility is to use MCMC methods to produce samples from a Markov chain having as stationary distribution the posterior of the model parameters, see e.g. Robert and Casella (2004). In the fully Bayesian approach, even MCMC algorithms can be inefficient and computationally demanding due to the structure of the likelihood.

In order to reduce the computational complexity of the fully Bayesian approach, in this paper we apply a combination of IFM and Bayesian procedures (shortly B-IFM), see e.g. Min and Czado (2010) and Dalla Valle, De Giuli, Tarantola, and Manelli (2016). Following Min and Czado (2010) and Czado and Min (2011), in a first step we use a frequentist approach to estimate the parameters $\boldsymbol{A}$ of the univariate marginal AR-GARCH models. This is done via the forward-backward approach implemented in the package rugarch of R. Subsequently, estimates of copula parameters $\Phi$ and of marginals parameters $\boldsymbol{v}$ (given the estimated parameters $\boldsymbol{A}$ ) are obtained in a Bayesian way. The posterior distributions for the copula parameters $\Phi$ and for the marginals parameters $\boldsymbol{v}$ (given the estimated parameters $\boldsymbol{A}$ ) can be obtained by

$\pi\left(\Phi, \boldsymbol{v} \mid \boldsymbol{A}, \mathcal{O}_{T}\right) \propto L\left(\mathcal{O}_{T} \mid \Phi, \boldsymbol{v}, \boldsymbol{A}\right) \pi(\Phi, \boldsymbol{v})$.

Although such a two steps procedure may lead to an underestimate of the uncertainty in the other parameters, in practice one usually does not see any significative difference between parameters estimated with a fully Bayesian approach and those estimated with this two steps approach. In Section 4 we compare the results obtained via our B-IFM procedures to the ones deriving from a fully Bayesian approach. Further comparisons are presented in the Supplementary Material.

In the following subsections, we describe the choice of the prior in the B-IFM setting for AR-GARCH copula models with Skew- $t$ marginals. The case of AR copula models and/or Normal marginals, as well as the fully Bayesian setting, can be obtained via straightforward modifications. More details are provided in Section 4 and in the Supplementary Material. It is important to observe that the Bayesian models that will be described in Sections 3.1-3.3, can be easily adapted to employ other types of copulas and/or marginal distributions.

\subsection{Bayesian tree copula model}

In this model, we assume that the standardised residuals have a tree copula distribution (3), where each $c_{\theta_{l, m}}$ is a Double Copula with parameter $\theta_{l, m}$, as described in Section 2.5. We suppose that the standardised residuals follow the univariate Hansen Skew$t$ pdfs with parameters $v_{k}=\left(\lambda_{k}, \eta_{k}\right)$ described in Section 2.4.

We assign the following independent prior distributions

$$
\begin{aligned}
\lambda_{k} & \sim \operatorname{Beta}_{(-1,1)}\left(\alpha_{k}, \beta_{k}\right) \\
\eta_{k} & \sim \operatorname{Exp}\left(\Lambda_{k}\right) \\
\theta_{l, m}=\left(\tau_{l, m}, \zeta_{l, m}\right) & \sim \operatorname{Beta}_{(-1,1)}\left(\delta_{l, m}, \gamma_{l, m}\right) \times \operatorname{Unif}_{\mathcal{H}}(\cdot) \\
\mathcal{E} & \sim \mathcal{U}(\cdot),
\end{aligned}
$$

for $k=1, \ldots, N$ and $(l, m) \in \mathcal{E}$. We denote with $\pi(\boldsymbol{v}, \boldsymbol{\theta}, \mathcal{E})$ the corresponding joint prior density. In $(4), \operatorname{Beta}_{(-1,1)}(\alpha, \beta)$ indicates a translated Beta distribution on $(-1,1)$ of parameters $(\alpha, \beta)^{1}$ and Unif $_{\mathcal{H}}(\cdot)$ is a uniform distribution on $\mathcal{H}=\left\{D G_{1}, D G_{2}, D C_{1}, D C_{2}\right\}$. For the prior on the degrees of freedom, following Geweke (1993), we consider an exponential distribution $\operatorname{Exp}(\Lambda)$ with parameter $\Lambda$. In the absence of specific prior information on the dependence structure, we use uniform prior on $\mathscr{E}_{N}$. Note that this prior is a special case of the default decomposable prior proposed by Meilă and Jaakkola (2006). The joint posterior density is

$$
\begin{gathered}
\pi\left(\boldsymbol{\theta}, \boldsymbol{v}, \mathcal{E} \mid \mathcal{O}_{T}, \boldsymbol{A}\right) \propto \prod_{t=1}^{T} \prod_{(l, m) \in \mathcal{E}} c_{\theta_{l, m}}\left(F_{v_{l}}\left(\frac{x_{l, t}-\sum_{i=1}^{p} a_{l, i} x_{l, t-i}}{\sigma_{l, t}}\right),\right. \\
\left.F_{v_{m}}\left(\frac{x_{m, t}-\sum_{i=1}^{p} a_{m, i} x_{m, t-i}}{\sigma_{m, t}}\right)\right) \\
\quad \times \prod_{k=1}^{N} \frac{1}{\sigma_{k, t}} f_{v_{k}}\left(\frac{x_{k, t}-\sum_{i=1}^{p} a_{k, i} x_{k, t-i}}{\sigma_{k, t}}\right) \pi(\boldsymbol{\theta}, \boldsymbol{v}, \mathcal{E}),
\end{gathered}
$$

where $\boldsymbol{\theta}$ is the collection of all the copula parameters $\theta_{l, m}$.

Since the previous posterior density cannot be obtained in closed form, we rely on a Metropolis within Gibbs algorithm, based on the works of Gruber and Czado (2014, 2015), Silva and Gramcy (2009). For more details, see the Supplementary Material.

\subsection{Bayesian tree copula mixture model}

We now consider the case in which the joint distribution of the standardised residuals is represented via a finite mixture of $D$ tree copulas with double linking copulas and Hansen Skew-t margins. The pdf of the standardised residuals is equal to

$$
\begin{aligned}
& f_{\mathbf{Z}_{t}}\left(z_{1, t}, \ldots, z_{N, t}\right) \\
& \quad=\sum_{d=1}^{D} w_{d} \prod_{(l, m) \in \mathcal{E}_{d}} c_{\theta_{l, m}^{(d)}}\left(F_{v_{l}}\left(z_{l, t}\right), F_{v_{m}}\left(z_{m, t}\right)\right) \prod_{k=1}^{N} f_{v_{k}}\left(z_{k, t}\right) .
\end{aligned}
$$

\footnotetext{
${ }^{1} X$ has translated Beta distribution on $(-1,1)$ with parameters $(\alpha, \beta)$, if $X=$ $2 Y-1$ where $Y$ has Beta distribution with parameters $(\alpha, \beta)$.
} 
In the previous equation, $\boldsymbol{w}=\left(w_{1}, \ldots, w_{D}\right)$ is the vector of weights and $\mathcal{E}_{d}$ and $\boldsymbol{\theta}_{d}=\left\{\theta_{l, m}^{(d)}\right\}$ are the tree copula structure and the vector of copula parameters for the $d$ th component, respectively. Finally, we set $\mathfrak{E}=\left\{\mathcal{E}_{1}, \ldots, \mathcal{E}_{D}\right\}$ and $\Theta=\left\{\boldsymbol{\theta}_{1}, \ldots, \boldsymbol{\theta}_{D}\right\}$.

We assign the following independent prior distributions to the parameters

$$
\begin{aligned}
\boldsymbol{w} & \sim \operatorname{Dir}\left(\psi_{1}, \ldots, \psi_{D}\right) \\
\lambda_{k} & \sim \operatorname{Beta}_{(-1,1)}\left(\alpha_{k}, \beta_{k}\right) \\
\eta_{k} & \sim \operatorname{Exp}\left(\Lambda_{k}\right) \\
\theta_{l, m}^{(d)}=\left(\tau_{l, m}^{(d)}, \zeta_{l, m}^{(d)}\right) & \sim \operatorname{Beta}_{(-1,1)}\left(\delta_{l, m}, \gamma_{l, m}\right) \times \operatorname{Unif}_{\mathcal{H}}(\cdot) \\
\mathcal{E}_{d} & \sim \mathcal{U}(\cdot)
\end{aligned}
$$

for $k=1, \ldots, N, d=1, \ldots, D$ and we denote with $\pi$ the corresponding prior density. In the above $\operatorname{Dir}\left(\psi_{1}, \ldots, \psi_{D}\right)$ is a Dirichlet distribution with density proportional to $\prod_{d=1}^{D-1} w_{d}^{\psi_{d}-1}(1-$ $\sum_{j=1}^{D-1} w_{j}$ ), with $w_{D}=1-\sum_{d=1}^{D-1} w_{d}$.

In order to sample from the posterior distribution of the finite mixture of tree copulas we adopt the data augmentation procedure by Van Dyk and Meng (2001). The details of the resulting Metropolis within Gibbs algorithm are described in the Supplementary Material.

\subsection{DP-tree copula model}

As an extension of the tree copula mixture model described in the previous subsection, we propose a Bayesian nonparametric approach based on a Dirichlet process (DP) mixture model. In this way we do not need to fix a priori the number of the mixture components.

The Dirichlet process $D P\left(G_{0}, \psi\right)$ is a measure on measures. It has two parameters, a scaling parameter $\psi>0$ and a base probability measure $G_{0}$. It was first formalised by Ferguson (1973) for general Bayesian statistical modelling, as a prior over distributions with wide support yet tractable posteriors. It is currently one of the most popular Bayesian nonparametric model. The so-called stick breaking construction, Sethuraman (1994), shows that if $G$ is a draw from a $\operatorname{DP}\left(G_{0}, \psi\right)$, then $G(\cdot)=\sum_{d \geq 1} w_{d} \delta_{\Psi_{d}}(\cdot)$ where the atoms $\Psi_{d}$ are i.i.d. random variables with distribution $G_{0}, \delta_{\Psi_{d}}$ is a probability measure concentrated on $\Psi_{d}$ and the weights $w_{d}$ are generated using the stick-breaking construction. More precisely, $w_{1}=v_{1}$ and $w_{d}=v_{d} \prod_{l<d}\left(1-v_{l}\right)$ with $v_{d}$ i.i.d. random variables with $\operatorname{Beta}(1, \psi)$ distribution.

The DP mixture model can be derived as the limit of a finite mixture model where the number of the components tends to infinity, see e.g. Ishwaran and Zarepour (2002), and allows for the inclusion of the the uncertainty about the number of components.

In the DP-Tree Copula Model we assume that the pdf of the standardised residuals at time $t$ has the form

$$
\begin{aligned}
f_{Z_{t}}\left(z_{1, t}, \ldots, z_{N, t}\right)= & \int \prod_{(l, m) \in \mathcal{E}} c_{\theta_{l, m}}\left(F_{v_{l}}\left(z_{l, t}\right), F_{v_{m}}\left(z_{m, t}\right)\right) \\
& \times \prod_{k=1}^{N} f_{v_{k}}\left(z_{k, t}\right) G(d \boldsymbol{\theta} d \mathcal{E}),
\end{aligned}
$$

where $G \sim D P\left(\psi, G_{0}\right)$. By the stick breaking representation we obtain

$$
\begin{aligned}
f_{Z_{t}} & \left(z_{1, t}, \ldots, z_{N, t}\right) \\
& =\sum_{d \geq 1} w_{d} \prod_{(l, m) \in \mathcal{E}_{d}} c_{\theta_{l, m}^{(d)}}\left(F_{v_{l}}\left(z_{l, t}\right), F_{v_{m}}\left(z_{m, t}\right)\right) \prod_{k=1}^{N} f_{v_{k}}\left(z_{k, t}\right) .
\end{aligned}
$$

The base measure $G_{0}$ decomposes into the product of a prior on $\boldsymbol{\theta}_{d}$ and a prior on $\mathcal{E}_{d}$. We also assume that the concentration parameter $\psi$ of the DP and the parameters $\boldsymbol{v}$ are unknown.
Summarising, in the DP-Tree Copula model the prior $\pi$ is described by

$$
\begin{aligned}
\lambda_{k} & \sim \operatorname{Beta}_{(-1,1)}\left(\alpha_{k}, \beta_{k}\right) \\
\eta_{k} & \sim \operatorname{Exp}\left(\Lambda_{k}\right) \\
v_{d} & \sim \operatorname{Beta}(1, \psi) \\
\left(\boldsymbol{\theta}_{d}, \mathcal{E}_{d}\right) & \sim G_{0}(\cdot) \\
\psi & \sim \operatorname{Gamma}\left(a_{\psi}, b_{\psi}\right)
\end{aligned}
$$

for $k=1, \ldots, N$ and $d \geq 1$. In (6), Gamma( $a, b)$ denotes a Gamma density with shape parameter $a$ and scale parameter $b$. Moreover, we assume that $G_{0}$ is equal to the product between a prior on $\boldsymbol{\theta}_{d}$ with density $p_{0}$ and a uniform prior on the tree structure $\mathcal{E}_{d}$. Since $\boldsymbol{\theta}_{d}=\left\{\theta_{l, m}^{(d)}\right\}$, we set $p_{0}=\prod_{(l, m)} \operatorname{Beta}_{(-1,1)}\left(\delta_{l, m}, \gamma_{l, m}\right)$.

In order to sample from the posterior distribution we adopt a variant of the slice sampling MCMC algorithm, proposed by Walker (2007) and Kalli, Griffin, and Walker (2011). See the Supplementary Material.

\subsection{Model comparison}

A commonly used criterion for model comparison is the Deviance Information Criterion (DIC) introduced by Spiegelhalter, Best, Carlin, and van der Linde (2002). Since DIC cannot be applied to mixture models, we rely on $D I C_{3}$, a variant of the original criterion, see Richardson (2002) and Celeux, Forbes, Robert, and Titterington (2006). Given a density function $f\left(\boldsymbol{x}_{1: T} \mid \boldsymbol{\varphi}\right)$ depending on a set of parameters $\varphi$, the DIC is defined as

$D I C=-4 \mathbb{E}_{\boldsymbol{\varphi}}\left[\log f\left(\boldsymbol{x}_{1: T} \mid \boldsymbol{\varphi}\right) \mid \boldsymbol{x}_{1: T}\right]+2 \log f\left(\boldsymbol{x}_{1: T} \mid \tilde{\boldsymbol{\varphi}}\right)$,

where $\tilde{\varphi}$ is a posterior estimate of $\varphi$ (a common choice is the posterior mean) and $\boldsymbol{x}_{1: T}=\left(\boldsymbol{x}_{1}, \ldots, \boldsymbol{x}_{T}\right)$ is the set of observations up to time $T$.

In the mixture model the set of parameters $\varphi$ is not always identifiable, and consequently we cannot obtain $\tilde{\varphi}$ straightforwardly. To overcome this problem, one can replace the term depending on $\tilde{\varphi}$ in Eq. (7) with a function invariant under permutation. A natural choice is to consider the posterior predictive $\mathbb{E}_{\boldsymbol{\varphi}}\left[f\left(\boldsymbol{x}_{1: T} \mid \boldsymbol{\varphi}\right) \mid \boldsymbol{x}_{1: T}\right]$, obtaining in this way the $\mathrm{DIC}_{3}$ as

$$
D I C_{3}=-4 \mathbb{E}_{\boldsymbol{\varphi}}\left[\log f\left(\boldsymbol{x}_{1: T} \mid \boldsymbol{\varphi}\right) \mid \boldsymbol{x}_{1: T}\right]+2 \log \left[\mathbb{E}_{\boldsymbol{\varphi}}\left[f\left(\boldsymbol{x}_{1: T} \mid \boldsymbol{\varphi}\right) \mid \boldsymbol{x}_{1: T}\right]\right] .
$$

Although these measures present some limitations (see the discussion of the paper of Spiegelhalter, Best, Carlin, and van der Linde (2002), they are simple to calculate using MCMC and therefore, despite the criticisms, widely used in the Literature.

As an alternative measure for model comparison, following the econometric Bayesian literature, we consider the cumulative logscore, see e.g. Gneiting and Raftery (2007). It is defined as

log-score $=\sum_{t=\tau_{0}}^{\tau_{1}} \log \left\{\mathbb{E}_{\boldsymbol{\varphi}}\left[f\left(\boldsymbol{x}_{t+1} \mid \boldsymbol{x}_{1: t}, \boldsymbol{\varphi}\right) \mid \boldsymbol{x}_{1: t}\right]\right\}$,

where $\boldsymbol{x}_{1: t}=\left(\boldsymbol{x}_{1}, \ldots, \boldsymbol{x}_{t}\right)$ is the set of observations up to time $t, \boldsymbol{\varphi}$ is the set of parameters, $f\left(\boldsymbol{x}_{t+1} \mid \boldsymbol{x}_{1: t}, \varphi\right)$ is the conditional likelihood and $\mathbb{E}_{\varphi}\left[f\left(\boldsymbol{x}_{t+1} \mid \boldsymbol{x}_{1: t}, \boldsymbol{\varphi}\right) \mid \boldsymbol{x}_{1: t}\right]$ is the posterior predictive distribution. Higher values of the log-score correspond to better fits.

\subsection{Dependence analysis}

For the tree copula model of Section 3.1 the dependence structure between the standardised residuals is directly encoded by the underling graphical structure $\mathcal{E}$. A simple estimate of the unknown tree structure $\mathcal{E}$ is the maximum a posteriori probability (MAP) tree structure defined as $\mathcal{E}_{M A P}=\operatorname{argmax}_{\mathcal{E}} \pi\left(\mathcal{E} \mid \mathcal{O}_{T}\right)$. In addition to 
the MAP tree one can evaluate the values of the Kendall's tau between pairs of standardised residuals. As an estimate of this measure we compute its predictive posterior mean

$$
\begin{aligned}
\hat{\tau}_{i, j} & =\mathbb{E}\left[\tau\left(z_{i, T+1}, z_{j, T+1} \mid \boldsymbol{\Phi}, \boldsymbol{v}\right) \mid \mathcal{O}_{T}\right] \\
& =\int \tau\left(z_{i, T+1}, z_{j, T+1} \mid \boldsymbol{\Phi}, \boldsymbol{v}, \boldsymbol{A}\right) \pi\left(d \boldsymbol{\Phi} d \boldsymbol{v} \mid \mathcal{O}_{T}, \boldsymbol{A}\right),
\end{aligned}
$$

where $\tau\left(z_{i, T+1}, z_{j, T+1} \mid \boldsymbol{\Phi}, \boldsymbol{v}, \boldsymbol{A}\right)$ is the Kendall's tau between the variables $z_{i, T+1}$ and $z_{j, T+1}$ under the AR-GARCH copula model given the unknown parameters $\boldsymbol{\Phi}, \boldsymbol{v}$ and the marginally estimated parameters $\boldsymbol{A}$. For the models described in Sections 3.1-3.3, numerical approximations of the previous quantities can be easily obtained from the output of the corresponding MCMC algorithm.

For the mixture of tree copula models, in order to obtain a representative graphical structure, we apply the Minimum Spanning Tree (MST) approach. This procedure allows us to obtain a representative tree from the weighted graph based on Kendall's tau, see e.g. Wang and Xie (2016). More precisely, we consider a complete weighted graph in which the weight of each edge $(i, j)$ is the absolute value of the (estimated) Kendall's tau between the variables $i$ and $j$, i.e. $\left|\hat{\tau}_{i, j}\right|$. The Minimum Spanning Tree is the spanning tree that maximises the sum of edge weights or, equivalently, it is the spanning tree $\mathcal{E}^{*}$ such that $\sum_{(i, j) \in \mathcal{E}^{*}}\left(1-\left|\hat{\tau}_{i, j}\right|\right)=$ $\min _{\mathcal{E}} \sum_{(i, j) \in \mathcal{E}}\left(1-\left|\hat{\tau}_{i, j}\right|\right)$, where the minimum is taken over the set of all possible spanning trees.

It is worth noticing that there is no direct correspondence between missing edges in the MST and conditional independences between the variables. In particular there is no connection between this tree and a possible Markov Tree structure associated to the joint distribution of the variables.

\subsection{Risk measures}

Different risk measures are usually used to analyse and quantify the tail risk exposure, see e.g. Klugman, Panjer, and Willmot (2008) and Szegö (2005). In order to evaluate the market risk of an energy portfolio we focus on two well-known quantile risk measures: Value-at-Risk (VaR) and Expected Shortfall (ES).

Following Artzner, Delbaen, Eber, and Heath (1999), the VaR at given probability level $\alpha$ is defined as

$\operatorname{VaR}_{\alpha}(V)=-\inf \left\{v: F_{V}(v) \geq \alpha\right\}$,

where $F_{V}$ is the cdf of the net worth $V$ of a portfolio and the inf or infimum of a set of numbers is its greatest lower bound. Typically $\alpha$ is set equal to 0.01 or 0.05 , corresponding to the so-called 99\% and 95\% VaR. Equivalently, VaR can be defined in terms of the distribution of the losses; the $99 \% \mathrm{VaR}$ is the 0.99 quantile of the loss distribution, corresponding to minus the 0.01 quantile of the net worth distributions. The VaR is one of the most commonly used risk measure and is easy to estimate. The $99 \% \mathrm{VaR}$ for a horizon of two weeks is acceptable measure of risk according to the Basel Committee on Banking and Supervision of Banks for International Settlement (Basel Committee on Banking Supervision (1995) and following amendments). Nevertheless, many authors have criticised its adequacy as a measure of risk for different reasons, see e.g. Acerbi and Tasche (2002). The main problems are the following. First of all, it considers only a single quantile of the portfolio distribution, so that it does not provide any information about the potential size of loss that exceeds its value. Secondly, it does not satisfy the sub-additivity property and, consequently, it may underestimate the portfolio risk. A measure that overcomes the previous problems is the Expected Shortfall $(E S)$, see eg Artzner, Delbaen, Eber, and Heath (1999). For a significance level $\alpha$, the $E S$ is (minus) the conditional expectation of $V$, given that $V$ is below $-\operatorname{VaR}_{\alpha}(V)$, i.e.

$E S_{\alpha}=-\mathbb{E}\left[V \mid V<-\operatorname{VaR}_{\alpha}(V)\right]$.
$E S$ is a coherent risk measure and, in contrast to VaR, is sensitive to the severity of losses beyond VaR. For a comprehensive and critical comparison between VaR and ES, see e.g. Embrechts, Puccetti, Rüschendorf, Wang, and Beleraj (2014) and Emmer, Kratz, and Tasche (2015).

Using a Bayesian approach, given the data observed until time $T$, the $k$-step-ahead $\operatorname{VaR}$ at level $\alpha$, i.e. the $\operatorname{VaR}_{\alpha}\left(V_{T+k}\right)$, can be estimated using the $\alpha$-quantile of the $k$-step-ahead (posterior) predictive distribution of the net worth. This posterior predictive distribution at time $T+k$ is given by

$$
\begin{aligned}
F_{V_{T+k}}\left(v \mid \mathcal{O}_{T}\right) & :=P\left\{V_{T+k} \leq v \mid \mathcal{O}_{T}\right\} \\
& =\int P\left\{V_{T+k} \leq v \mid \mathcal{O}_{T}, \boldsymbol{\Phi}, \boldsymbol{v}\right\} \pi\left(d \boldsymbol{\Phi} d \boldsymbol{v} \mid \mathcal{O}_{T}\right) .
\end{aligned}
$$

In the previous equation, $P\left\{V_{T+k} \leq v \mid \mathcal{O}_{T}, \boldsymbol{\Phi}, \boldsymbol{v}\right\}$ is the predictive distribution of $V_{T+k}$ given the observations $\mathcal{O}_{T}$ and the unknown parameters $\boldsymbol{\Phi}, \boldsymbol{v}$, and $\pi\left(d \boldsymbol{\Phi} d \boldsymbol{v} \mid \mathcal{O}_{T}\right)$ is the posterior distribution of the parameters given the observations $\mathcal{O}_{T}$. Hence, $\operatorname{VaR}_{\alpha}\left(V_{T+k}\right)$, is estimated by

$V_{a R_{\alpha, T+k}}=-\inf \left\{v: F_{V_{T+k}}\left(v \mid \mathcal{O}_{T}\right) \geq \alpha\right\}$

and the $k$ step ahead ES at level $\alpha$ is estimated by

$E S_{\alpha, T+k}=-\mathbb{E}\left[V_{T+k} \mid V_{T+k}<-V a R_{\alpha, T+k}, \mathcal{O}_{T}\right]$.

The previous quantities can be easily approximated using the MCMC output, see e.g. Osiewalski and Pajor (2010).

\section{Empirical analysis}

\subsection{Data description}

We apply the proposed models to the analysis of the Italian and German energy markets. We consider the following variables: Power (Italy/German), Brent, TTF (natural gas price deriving from transactions in virtual trading points in the Netherlands), $\mathrm{CO}_{2}$ (price to pay for the emission of carbon dioxide into the atmosphere) and API (index representing a reference price benchmark for coal imported into North-West Europe). For the Italian market we also take into account PSV (natural gas price deriving from transactions in virtual trading points in Italy). The data are daily observations of one-year forward contracts. Power prices are obtained from the European Energy Exchange $(\mathrm{EEX})^{2}$, the leading energy exchange in Central Europe, while the remaining data are obtained from Reuters ${ }^{3}$; all values are expressed in Euros. Due to the intrinsic nature of these contracts, the dependence structure among the considered variables can differ significantly from one year to another. Since our model does not take into account changes of regime, in our analysis we focus on a single year at the time. In the following we present a detailed analysis of the behaviour of Italian and German markets from January 2014 to December 2014. For completeness of the analysis in Section 4.3 we present a multi-year analysis of the Italian market. As described in Section 2, in our analysis we work with the monthly logarithmic return rates $X_{t, i}=\log \left\{S_{t+20, i} / S_{t, i}\right\}$ where $S_{t, i}$ is the price at each day $t$ of commodity $i$ and $t$ assumes values in the set of all working days. Descriptive measures for the considered commodities and for the corresponding logarithmic returns are reported in Table 1.

\subsection{Prior settings and models comparison}

In order to describe the marginal time series, we considered a variety of AR-GARCH models of different orders with Normal or

\footnotetext{
2 https://www.eex.com. ${ }^{3}$

http://www.reuters.com.
} 
Table 1

Descriptive statistics for the examined commodities (daily prices) and the corresponding (monthly) logarithmic returns (from January 2014 to December 2014).

\begin{tabular}{|c|c|c|c|c|c|c|c|}
\hline & \multicolumn{7}{|c|}{ Commodities } \\
\hline & $\begin{array}{l}\text { Power } \\
\text { Italy }\end{array}$ & $\begin{array}{l}\text { Power } \\
\text { Germany }\end{array}$ & Brent & API & TTF & PSV & $\mathrm{CO}_{2}$ \\
\hline Mean & 53.71 & 35.07 & 72.84 & 58.82 & 25.77 & 27.47 & 6.095 \\
\hline Std & 1.69 & 0.82 & 6.59 & 1.63 & 0.92 & 0.86 & 0.63 \\
\hline Min & 49.80 & 33.77 & 50.67 & 54.68 & 23.05 & 24.81 & 4.48 \\
\hline Max & 58.45 & 36.90 & 79.66 & 63.85 & 27.81 & 29.10 & 7.42 \\
\hline Kurt & 3.67 & 2.31 & 6.94 & 2.43 & 2.89 & 2.70 & 2.32 \\
\hline \multirow[t]{3}{*}{ Skew } & 0.86 & 0.64 & -1.97 & -0.55 & -0.20 & -0.38 & -0.13 \\
\hline & \multicolumn{7}{|c|}{ Logarithmic return rates } \\
\hline & $\begin{array}{l}\text { Power } \\
\text { Italy }\end{array}$ & $\begin{array}{l}\text { Power } \\
\text { Germany }\end{array}$ & Brent & API & TTF & PSV & $\mathrm{CO}_{2}$ \\
\hline Mean & -0.34 & -0.13 & -0.37 & -0.25 & -0.58 & 0.19 & -0.28 \\
\hline Std & 0.022 & 0.023 & 0.028 & 0.030 & 0.027 & 0.131 & 0.062 \\
\hline Min & -3.18 & -3.07 & -2.84 & -2.60 & -2.59 & -3.74 & -3.83 \\
\hline Max & 2.02 & 2.15 & 2.42 & 2.61 & 1.47 & 2.76 & 0.78 \\
\hline Kurt & 3.46 & 2.99 & 3.27 & 3.44 & 2.08 & 4.90 & 6.20 \\
\hline Skew & -0.32 & -0.17 & 0.22 & 0.20 & -0.080 & -0.59 & -1.80 \\
\hline
\end{tabular}

Skew- $t$ marginal distributions. Following a frequentist approach we selected the model and the order that best fits the data through the AIC criterion. Table 2 lists the AIC values for a set of representative cases.

We note that none of the models has the lowest AIC value for all marginals. On the other hand, we observe that the GARCH model shows the worst fit and it will not be further investigated. We select $\operatorname{AR}(3)-\operatorname{GARCH}(1,1)$ and $\operatorname{AR}(3)$ models that show a good fit for a high number of marginal series.

In the following, we apply the Bayesian models described in Sections 3.1-3.3: the tree copula model (TCM), the finite mixture of tree copulas model (Mix-TCM), and the DP-tree copula model (DP-TCM). In addition to the Skew- $t$ margins case, for the sake of comparison, we also consider standard Normal marginal distributions. If an AR model is examined, we include in the parameters list also the precision $p_{k}=1 / \sigma_{k}^{2}$, see (1). This means that we assume $v_{k}=\left(\lambda_{k}, \eta_{k}, p_{k}\right)$ for Skew- $t$ margins and $v_{k}=p_{k}$ for Normal margins. Finally, for both cases we assume a standard Gamma prior for $p_{k}$.

For the prior distributions we consider the following hyperparameters values. We choose $\left(\delta_{l, m}, \gamma_{l, m}\right)=(1,1)$, i.e. a uniform prior for each $\theta_{l, m}$. In the finite mixture models we use a symmetric Dirichlet prior with hyperparameters equal to 10 for the weights $\mathbf{w}$. In DP-tree copula model the hyperparameters of the Gamma prior on $\psi$ are $\left(a_{\psi}, b_{\psi}\right)=(16,0.25)$. In case of marginal Skew- $t$ distribution we consider a translated Beta prior with parameters $(1,1)$ on $\lambda_{k}$ (skewness parameter) and an Exponential prior on $\eta_{k}$ (degree of freedom) with mean 10 . On the parameters precision $p_{k}$ we assign a Gamma prior with parameters $(1,1)$. Sensitivity analysis shows that the choice of the prior settings does not affect significantly the posterior estimates. The $\mathrm{DIC}_{3}$ is slightly sensitive to the choice of the hyperparameters for the prior on $\psi$. Hence we tuned them to get the best result in term of $\mathrm{DIC}_{3}$.

In Table 3 we compare the alternative models in terms of $\mathrm{DIC}_{3}$. The DP tree model with Skew- $t$ margins shows the best results for both the Italian and the German markets. Moreover, the models with Skew- $t$ margins are always better than the corresponding ones with Normal margins. Log-scores, computed out of sample on the last 50 observations and in sample on the last 200 observations, confirm these findings. In Fig. 2, we report the comparison among the out of sample cumulative log-score obtained with the DP-tree models applied to the $\operatorname{AR}(3)-\operatorname{GARCH}(1,1)$ and $\operatorname{AR}(3)$ estimated standardised residuals for each domestic market.
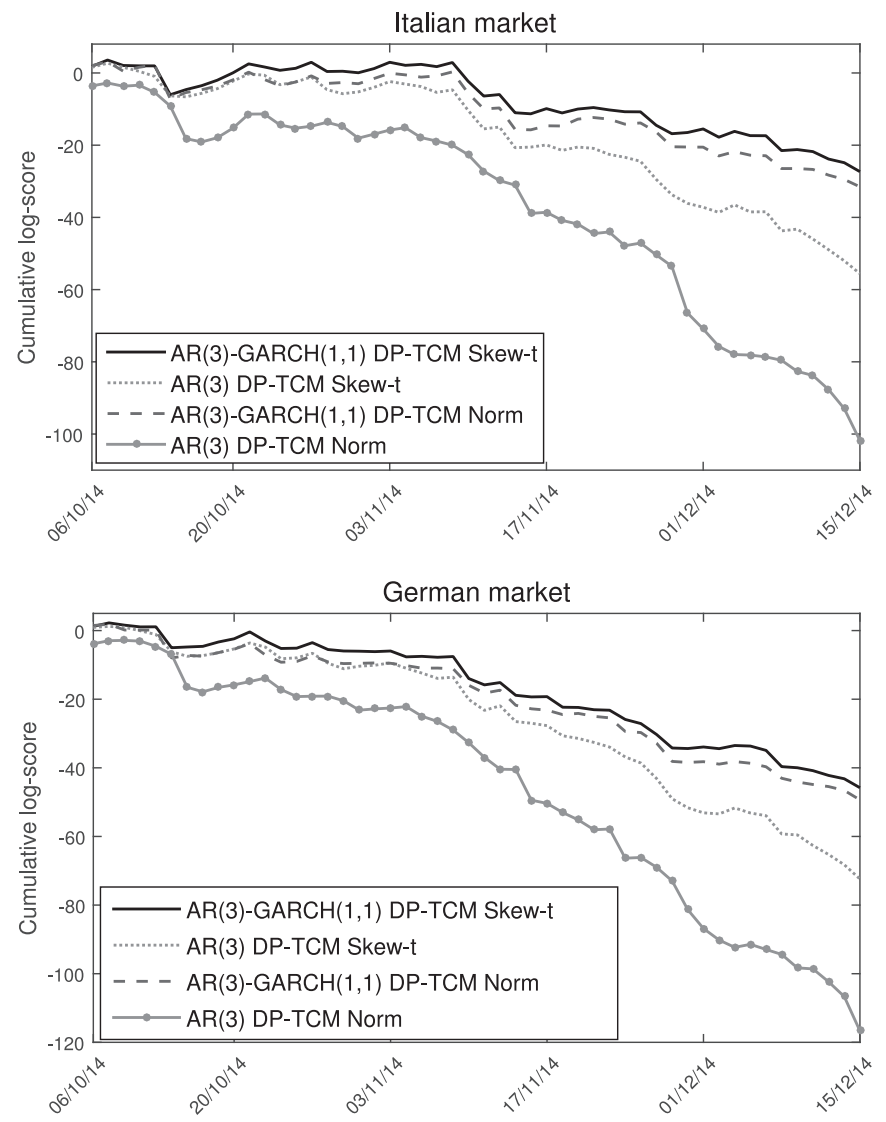

Fig. 2. Out of sample cumulative log-score (on the last 50 observations) for the DP-tree models: Italian market (first row) and German market (second row).

With regard to the marginal distribution of the standardised residuals, we computed the posterior mean and 95\% credible interval for the parameters $(\lambda, \eta)$ of the Skew-t. Table 4 shows the results for the DP-TCM. One can observe that the estimates of the skewness parameters $\lambda_{k}$ are very close to zero for all standardised residuals. The 0.95 credible intervals suggest that there is posterior support for zero for all commodities, although the ones associated with $\mathrm{CO}_{2}$ and Brent have zero very close to one of the end points. On the basis of the posterior means of $\lambda_{k}$, one can suppose that the standardised residuals of the $\mathrm{CO}_{2}$ series are slightly positively skewed, while Brent's standardised residuals are slightly negatively skewed. As far as the estimated degrees of freedom is concerned, the tails of the API standardised residuals are the most close to Normal (with the estimates of $\eta$ between 44 and 49 in the AR copula models and between 17 and 18 in the AR-GARCH copula models). For all the other commodities the estimated degrees of freedom range between 4 and 14, supporting the hypothesis of heavy tails.

As discussed in Section 3.3, using the DP-tree copula models one can also estimate the posterior distribution of the number of clusters. With the AR(3)-GARCH(1,1) DP-tree the posterior mode of the number of clusters is 6 for the Italian market and 8 for the German market. We have similar results with the AR(3) DP-tree copula model for which the posterior mode of the number of clusters is 5 for the Italian and 8 for the German one. The posterior histograms of the number of clusters are reported in Figs. S5 and S6 in the Supplementary Material.

\subsection{Dependence structure analysis}

In this subsection we study the dependence between the commodities in each domestic market. We start by considering the tree 
Table 2

AIC values for representative alternative models for the marginal series.

\begin{tabular}{llllllll}
\hline & PowIT & PowDE & TTF & PSV & API & CO $_{2}$ & Brent \\
\hline AR(2)-GARCH(2,2) Norm & 140 & 131 & 312 & 263 & 67 & 75 & -147 \\
AR(2)-GARCH(2,2) Skew- $t$ & 112 & 132 & 299 & 250 & 69 & 73 & -147 \\
AR(3)-GARCH(1,1) Norm & 141 & $\mathbf{1 3 1}$ & 307 & 255 & 63 & 75 & -147 \\
AR(3)-GARCH(1,1) Skew- $t$ & $\mathbf{1 1 2}$ & 132 & 293 & 243 & 66 & $\mathbf{6 7}$ & $\mathbf{- 1 4 8}$ \\
AR(3) Norm & 144 & 134 & 303 & 252 & $\mathbf{6 0}$ & 144 & -73 \\
AR(3) Skew- $t$ & 118 & 134 & $\mathbf{2 9 0}$ & $\mathbf{2 4 0}$ & 64 & 96 & -107 \\
AR(2) Norm & 142 & 132 & 304 & 257 & 60 & 140 & -75 \\
AR(2) Skew- $t$ & 115 & 132 & 291 & 245 & 63 & 94 & -110 \\
GARCH(1,1) Norm & 469 & 498 & 517 & 510 & 532 & 390 & 218 \\
GARCH(1,1) Skew- $t$ & 449 & 491 & 519 & 504 & 483 & 369 & 204 \\
\hline
\end{tabular}
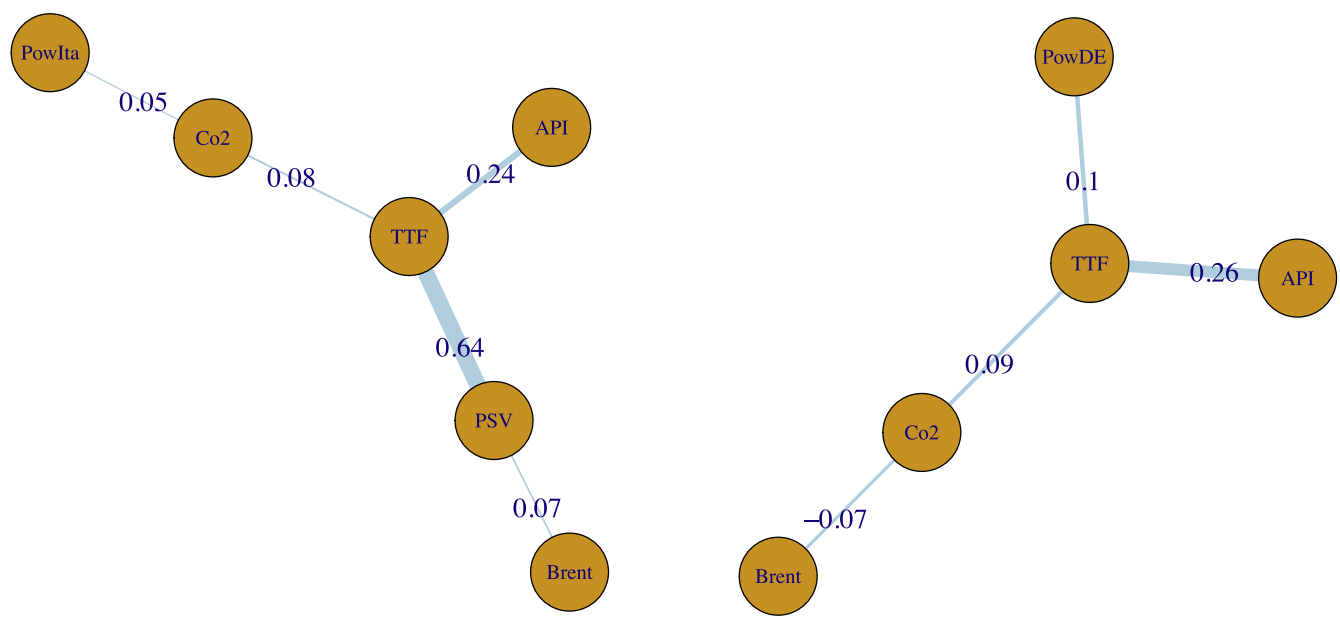

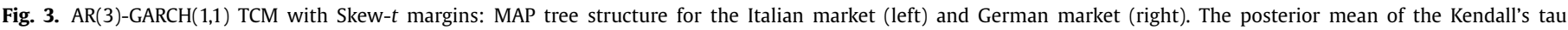
(given the MAP tree structure) is reported on each edge.

Table 3

$\mathrm{DIC}_{3}$ values for the alternative models.

\begin{tabular}{llllll}
\hline & \multicolumn{2}{l}{ Italy } & & & \multicolumn{2}{l}{ Germany } \\
\cline { 2 - 3 } & Norm & Skew- $t$ & & Norm & Skew- $t$ \\
\hline AR(3) TCM & 654 & 412 & & 655 & 365 \\
AR(3) Mix-TCM 2 Comp. & 624 & 367 & & 645 & 367 \\
AR(3) Mix-TCM 5 Comp. & 638 & 373 & & 656 & 376 \\
AR(3) DP-TCM & 564 & 341 & & 625 & 354 \\
AR(3)-GARCH(1,1) TCM & 235 & 217 & & 291 & 273 \\
AR(3)-GARCH(1,1)Mix-TCM 2 Comp. & 235 & 216 & & 287 & 276 \\
AR(3)-GARCH(1,1)Mix-TCM 5 Comp. & 237 & 237 & & 291 & 287 \\
AR(3)-GARCH(1,1) DP-TCM & 223 & 207 & & 281 & 270 \\
\hline
\end{tabular}

copula model with Skew- $t$ margins described in Section 3.1 applied to the estimated standardised residuals of the $\operatorname{AR}(3)-\operatorname{GARCH}(1,1)$ and of the $\operatorname{AR}(3)$ models. The posterior distribution on tree structures is quite flat and the probabilities of the MAP trees ranges between 0.08 and 0.22 (in the alternative models/markets). As an example, in Fig. 3 we report the MAP structures obtained for the $\operatorname{AR}(3)-G A R C H(1,1)$ model for each domestic market, with posterior probabilities equal to 0.22 and 0.13 , respectively. In Fig. 5 we display the corresponding results obtained with the $\operatorname{AR}(3)$ estimated standardised residuals. In this case, the posterior probabilities are 0.09 for the Italian market and 0.08 for the German market.

Ranking the trees with respect to their posterior probabilities, we find that the highest ranked trees share some interesting features. In order to obtain a cumulative posterior probability at least equal to 0.5 , in the $A R(3)-G A R C H(1,1)$ model we need to consider 6 structures for the Italian market and 8 structures for the German one. In all these structures Brent is an end node. Moreover, in the Italian case, Power Italy is always connected to TTF or $\mathrm{CO}_{2}$

Table 4

Posterior mean and 95\% credible interval (CI) for the parameters $(\eta, \lambda)$ obtained with the DP-TCM with Skew- $t$ margins.

\begin{tabular}{|c|c|c|c|c|c|c|c|c|c|}
\hline \multirow[t]{2}{*}{ Market } & & \multicolumn{4}{|c|}{ AR(3)-GARCH(1,1) marginal } & \multicolumn{4}{|c|}{$\mathrm{AR}(3)$ marginal } \\
\hline & & $\eta$ & $95 \% \mathrm{CI}$ & $\lambda$ & $95 \% \mathrm{CI}$ & $\eta$ & $95 \% \mathrm{CI}$ & $\lambda$ & $95 \% \mathrm{CI}$ \\
\hline \multirow[t]{6}{*}{ Italy } & PowIT & 4.69 & {$[3.31,7.29]$} & 0.03 & {$[-0.12,0.21]$} & 6.4 & {$[3.33,14.0]$} & 0.03 & {$[-0.11,0.17]$} \\
\hline & PSV & 7.81 & {$[4.49,15.02]$} & 0.05 & {$[-0.08,0.20]$} & 7.9 & {$[4.24,13.3]$} & -0.04 & {$[-0.27,0.13]$} \\
\hline & TTF & 4.82 & {$[3.39,7.65]$} & 0.04 & {$[-0.07,0.18]$} & 7.8 & {$[4.63,12.1]$} & 0.03 & {$[-0.16,0.19]$} \\
\hline & API & 17.7 & {$[9.1,27.9]$} & -0.06 & {$[-0.22,0.10]$} & 44.0 & {$[25.7,64.0]$} & -0.04 & {$[-0.21,0.11]$} \\
\hline & $\mathrm{CO}_{2}$ & 4.71 & {$[3.43,7.42]$} & 0.12 & {$[-0.03,0.27]$} & 4.11 & {$[2.80,6.41]$} & 0.136 & {$[-0.01,0.30]$} \\
\hline & Brent & 11.5 & {$[6.33,19.32]$} & -0.16 & {$[-0.33,0.01]$} & 3.54 & {$[2.25,5.91]$} & -0.10 & {$[-0.24,0.04]$} \\
\hline \multirow{5}{*}{ German } & PowDE & 13.58 & {$[9.70,27.2]$} & 0.10 & {$[-0.09,0.26]$} & 12.28 & {$[4.30,20.8]$} & 0.06 & {$[-0.12,0.24]$} \\
\hline & TTF & 4.54 & {$[3.20,7.31]$} & -0.03 & {$[-0.1,0.13]$} & 7.8 & {$[3.35,15.3]$} & -0.03 & {$[-0.19,0.11]$} \\
\hline & API & 18.6 & {$[6.7,34.7]$} & -0.05 & {$[-0.21,0.13]$} & 49.1 & {$[26.6,73.1]$} & -0.04 & {$[-0.22,0.15]$} \\
\hline & $\mathrm{CO}_{2}$ & 4.38 & {$[3.53,6.23]$} & 0.12 & {$[-0.01,0.30]$} & 4.00 & {$[2.84,6.11]$} & 0.14 & {$[0.01,0.30]$} \\
\hline & Brent & 12.5 & {$[6.33,18.9]$} & -0.16 & {$[-0.28,0.05]$} & 3.90 & {$[2.83,6.04]$} & -0.09 & {$[-0.22,0.04]$} \\
\hline
\end{tabular}



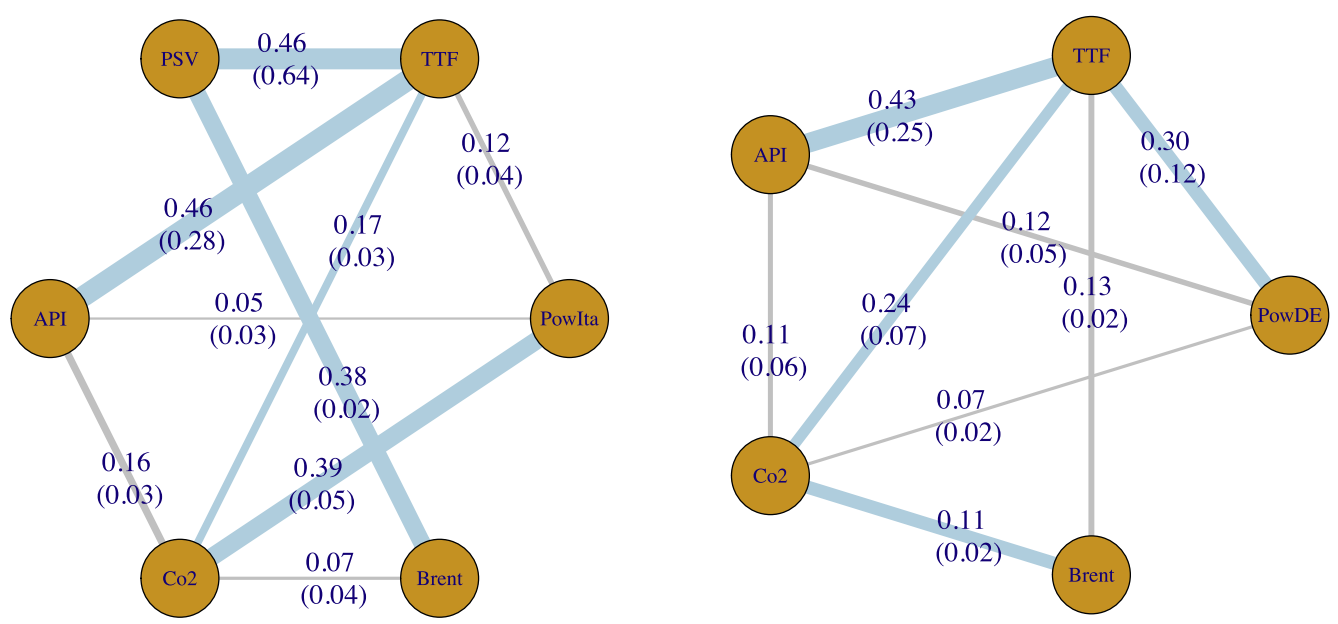

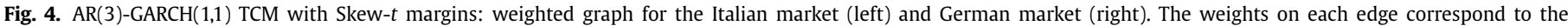

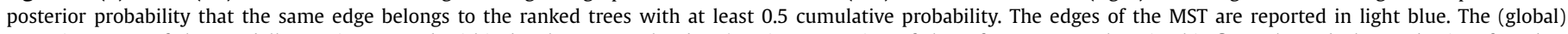

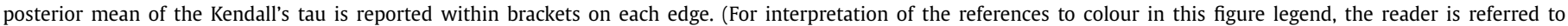
the web version of this article.)
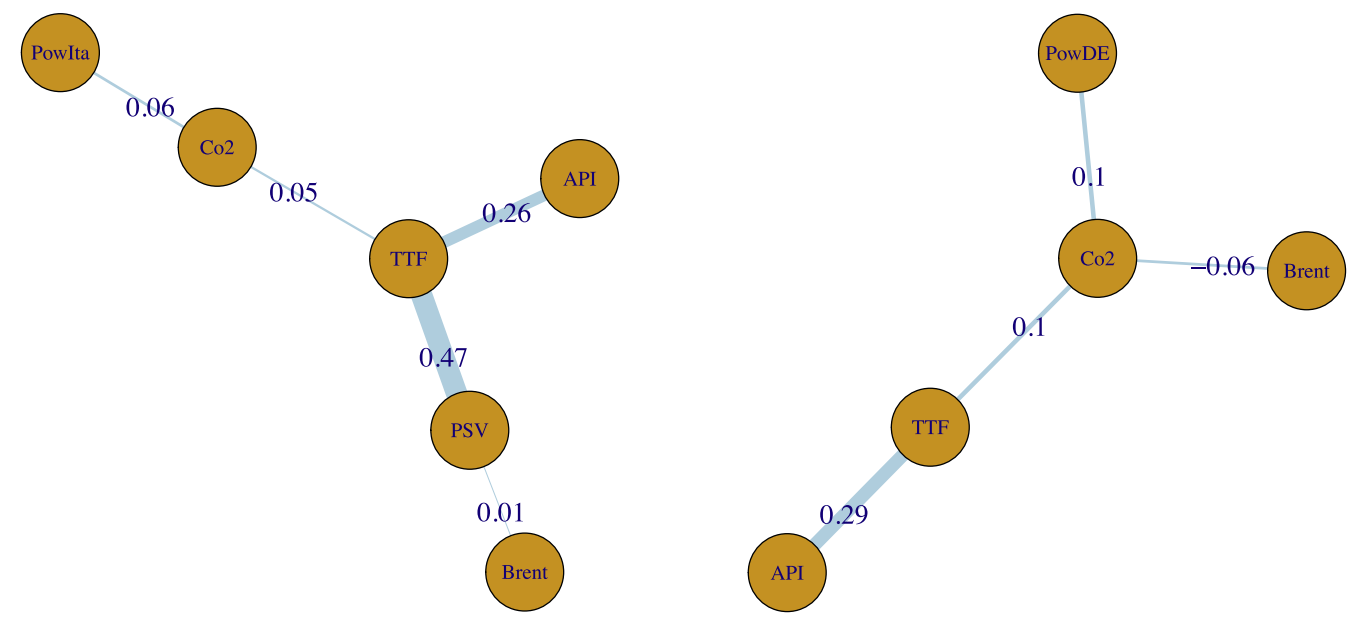

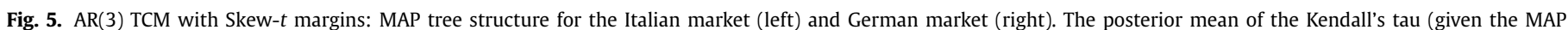
tree structure) is reported on each edge.

and, in addition, the path PSV-TTF-API is always included. For the German market, in each of these structures we find the edge TTFAPI and Power Germany is connected to TTF or $\mathrm{CO}_{2}$. In case of $\mathrm{AR}(3)$ estimated standardised residuals, we need to consider the first 8 ranked structures for the Italian market and the first 10 for the German market in order to obtain a cumulative posterior probability at least equal to 0.5 . Also in this case, for the Italian market we find the path PSV-TTF-API and Power Italy is connected to $\mathrm{CO}_{2}$. In the German market there are always the edges TTFAPI and Power Germany- $\mathrm{CO}_{2}$ and Brent is an end node in both markets.

The ranked trees are in line with some well-known characteristics of the current energy markets. Indeed, in both markets, Brent is always an end node and the value of the Kendall's tau of the corresponding edge is quite low confirming that the use of this commodity for energy production has drastically decreased in the last years. Moreover, as expected, the two gas nodes, TTF and PSV, are always connected in the Italian market and present high values of the Kendall's tau. The commodity that impacts mostly on the energy price is TTF, although also $\mathrm{CO}_{2}$ plays an important role in the market. This is consistent with the fact that TTF is one of the main raw material used for energy production and the cost of $\mathrm{CO}_{2}$ permissions cannot be neglected in the analysis of energy price behaviour, see e.g. Marimoutou and Soury (2015) and references therein.

Starting from the ranked tree structures, we constructed a graph with weights associated to each edge corresponding to the posterior probability that the same edge belongs to the ranked trees with at least 0.5 cumulative probability. Finally, we built the corresponding MST by maximising the posterior probabilities. The results are reported in Figs. 4 and 6. The edges defining the MST are in light blue. The grey edges are those not included in the MST. Edges with weight $<0.02$ are not depicted. We note that the MST structures are equal to the corresponding MAP trees, suggesting that the above discussed dependence paths are meaningful.

Following Section 3.5, we estimated pairwise Kendall's tau between the standardised residuals by computing the corresponding posterior mean for all the models, i.e. TCM, Mix-TCM and DPTCM. The estimated values are then used to construct the weighted graphs and the MST structures. In Figs. 7 and 8 we show the results for DP-TCM with Skew- $t$ margins applied to the estimated standardised residuals of the $\operatorname{AR}(3)-\operatorname{GARCH}(1,1)$ and $\operatorname{AR}(3)$. We can observe that some values of the Kendall's tau are very small. This is not surprising since we are dealing with the residuals of logreturns and not directly with prices of the commodities. In order to check that these small values are not due to mis-specification 

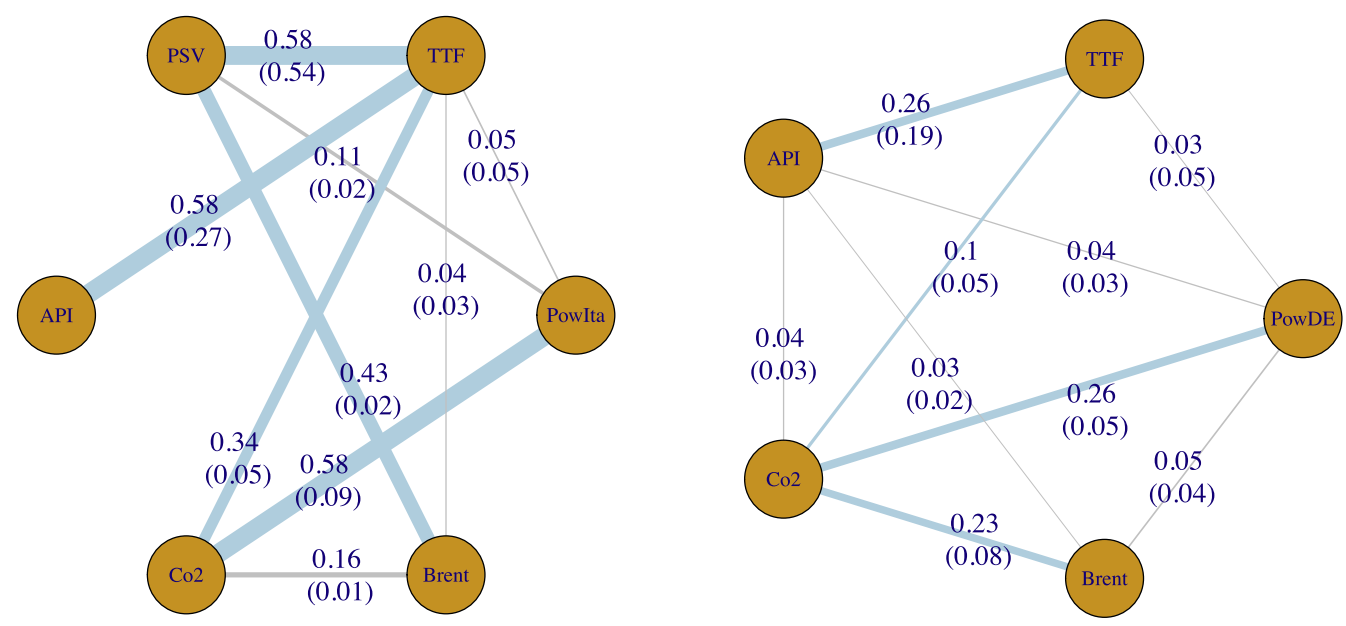

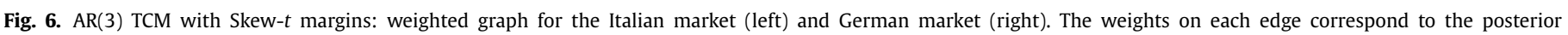

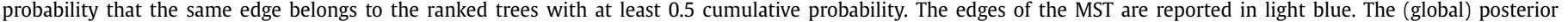

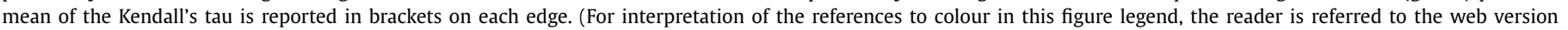
of this article)
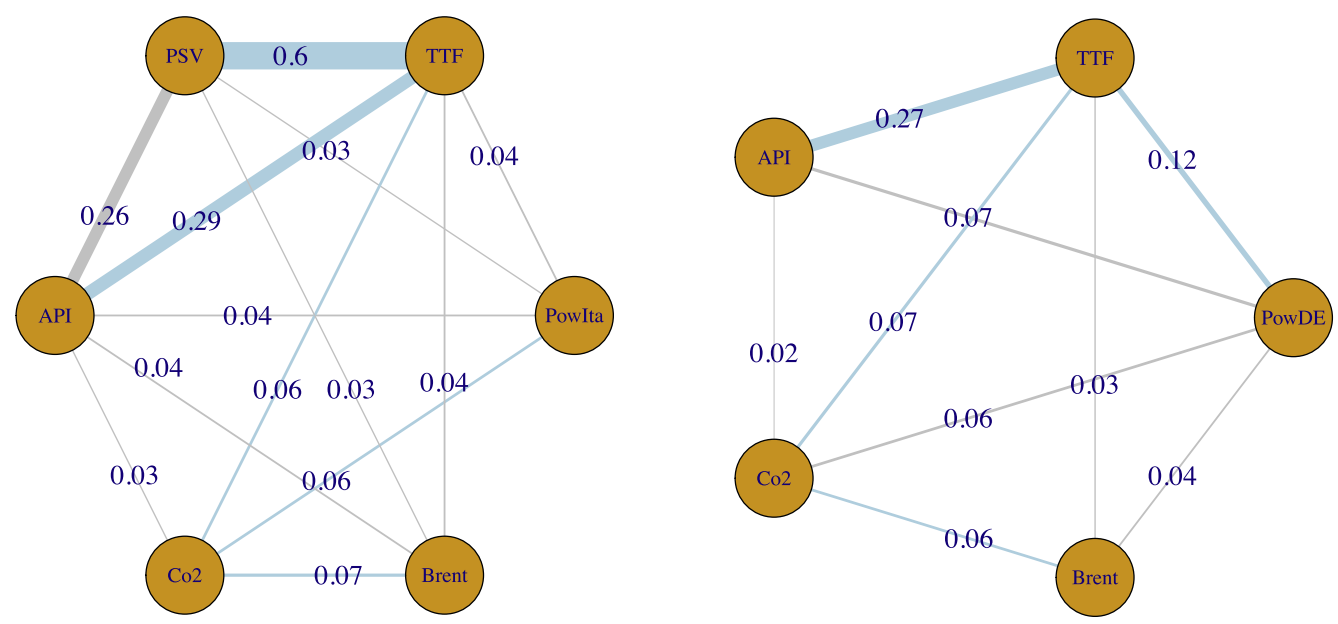

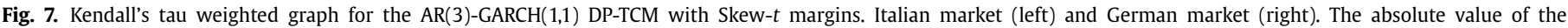

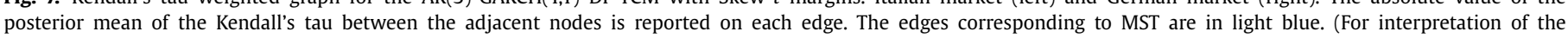
references to colour in this figure legend, the reader is referred to the web version of this article.)
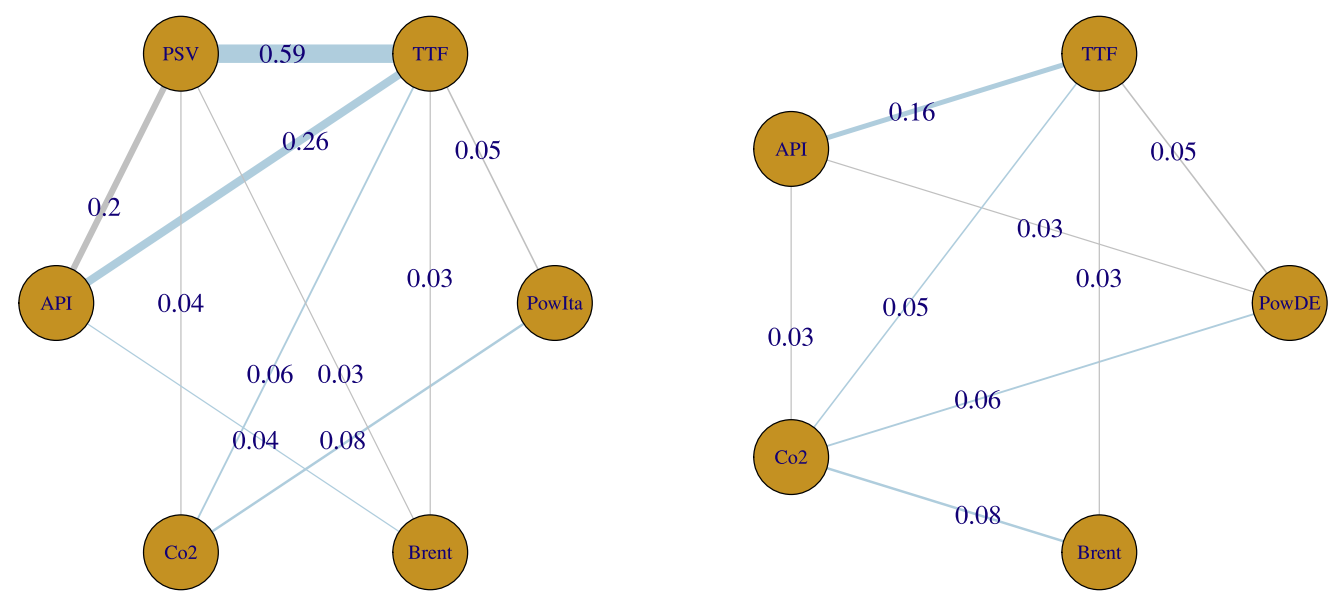

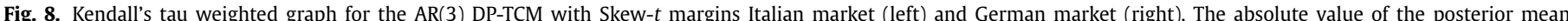

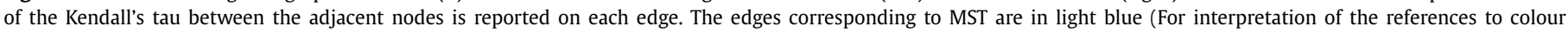
in this figure legend, the reader is referred to the web version of this article.). 

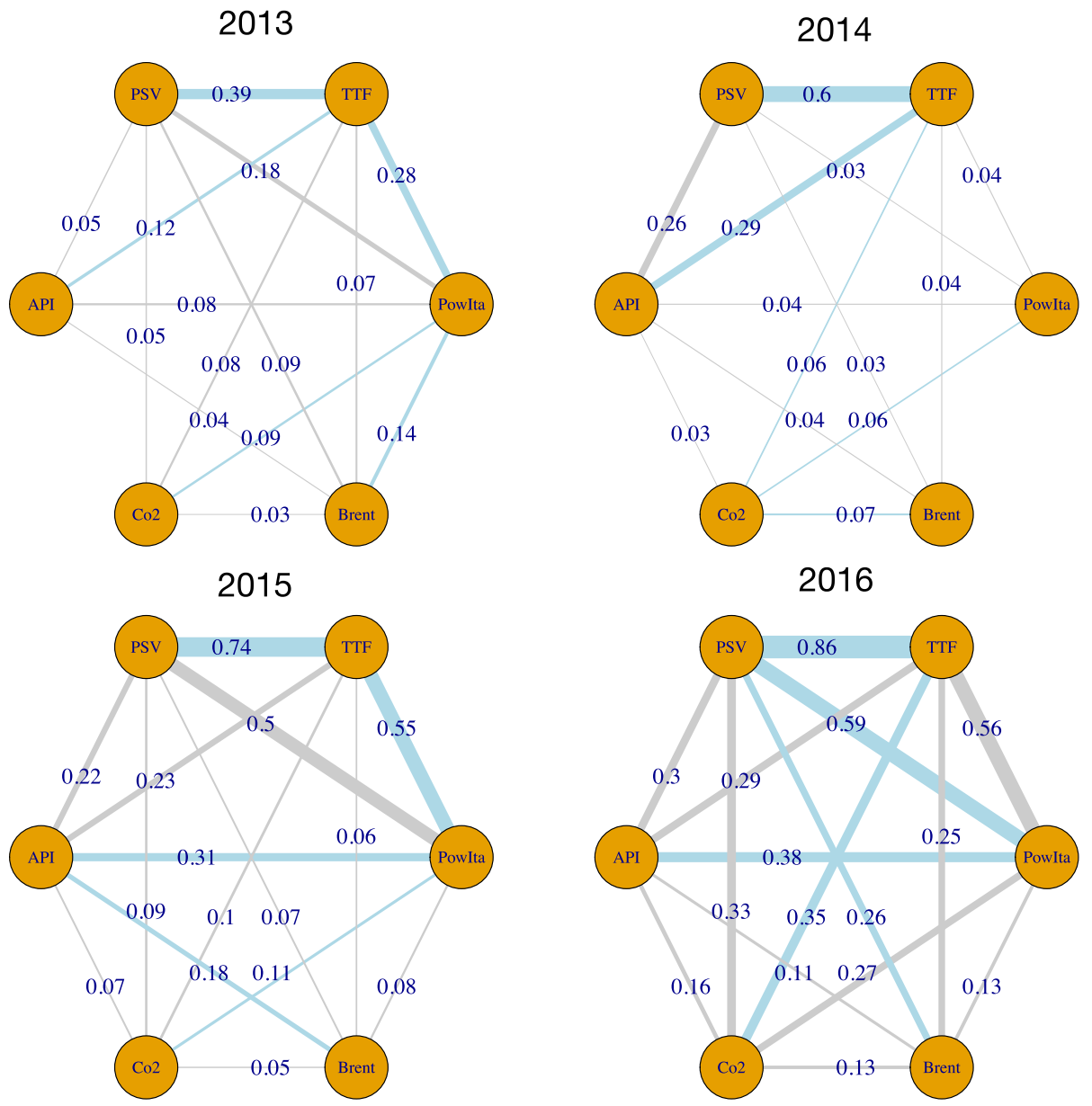

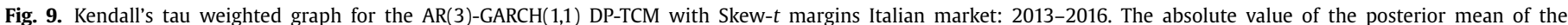

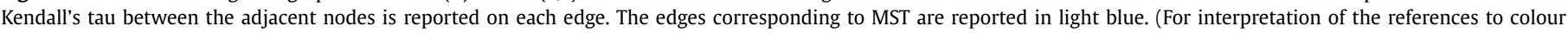
in this figure legend, the reader is referred to the web version of this article.)

of the linking copulas or to our estimation procedure, we also estimated these quantities by frequentist approach obtaining comparable results. Also a graphical comparison of the behaviour of the predictive residuals (obtained with our Bayesian model) with respect to the residuals of a frequentist IFM model suggests similar conclusions.

Comparing the MST structures for the DP-TCM models (Figs. 7 and 8) and the MAP trees for the TCM models (Figs. 3 and 5) we can note some similarities. For the German market the MST and the MAP tree structures are the same. On the other hand, for the Italian market they differ by the edge connecting Brent to the remaining part of each tree. In all structures Brent is an end point node with different neighbour sets. More precisely, in the MAPs it is adjacent to PSV, in the $\operatorname{AR}(3)-\operatorname{GARCH}(1,1)$ MST it is connected to $\mathrm{CO}_{2}$ and in the AR(3) MST is linked to API. All these edges present very low values of the estimated Kendall's tau.

We conclude this section with some results regarding a multiyear analysis of the Italian market. Italian energy contracts were traded for the first time by EEX in 2014. Here, we examine data from the previous year 2013 up to December 2016. Data for 2013 have been provided by traders of a leading Italian energy company. In Fig. 9 we report, for each year, the weighted graph based on the absolute values of the Kendall's tau obtained with the AR(3)$\operatorname{GARCH}(1,1)$ DP-TCM. It is interesting to note that the estimated values of the pairwise Kendall's tau increases for almost all variables from 2013 to 2016. More precisely, Kendall's tau between PSV and TTF increases from 0.37 to 0.86 , reflecting the reduction of price differential between PSV and TTF from 2013 to 2016, see the annual reports of Italian Regulatory Authority for Electricity and Gas (http://www.autorita.energia.it). An analogous behaviour characterises the dependence between API and Power Italy as well TTF/PSV and Power Italy. In contrast, Kendall's tau between Power Italy and Brent does not present an increasing trend, ranging between 0.02 and 0.13 . This can be possibly explained by the minor rule of Brent in the energy production.

\subsection{Energy market portfolio analysis}

In order to evaluate the market risk of an energy portfolio, we compute the Bayesian predictive $V a R$ and ES. For each domestic market, we consider a portfolio made of one power asset and the remaining examined commodities. In our analysis, we work in the perspective of an energy company that sells energy and buys the other commodities in order to produce it. Consequently, in the portfolio composition, power has a positive weight, $q_{1}$, while the remaining components should have negative weights, $-q_{i}, i=2, \ldots, N$. Hence, the portfolio value (corresponding to its net worth) at time $t$ is given by $V_{t}=q_{1} S_{t, 1}-\sum_{i=2}^{N} q_{i} S_{t, i}$. The composition of each portfolio has been provided us by experts of one of the major Italian energy company. More precisely, for the Italian market we consider a portfolio made of Power Italy, TTF, PSV, $\mathrm{API}, \mathrm{CO}_{2}$, Brent with weights $[1,0,0.14,0.28,0.69,0]$, while for the German one, we use a portfolio made of Power Germany, TTF, API, $\mathrm{CO}_{2}$, Brent with weights $[1,0.61,0.27,0.76,0]$. The weights are a good approximation to the real ones used by the energy company. In the following we refer to this portfolio as the "realistic" one. 
Table 5

MAEs between the out of sample forecasting portfolio (on the last 50 observations) and the historical portfolio.

\begin{tabular}{|c|c|c|c|c|c|c|c|c|}
\hline & \multicolumn{4}{|c|}{ "Realistic" portfolio } & \multicolumn{4}{|c|}{ Equally weighted portfolio } \\
\hline & \multicolumn{2}{|l|}{ Italy } & \multicolumn{2}{|c|}{ Germany } & \multicolumn{2}{|l|}{ Italy } & \multicolumn{2}{|c|}{ Germany } \\
\hline & Norm & Skew- $t$ & Norm & Skew- $t$ & Norm & Skew- $t$ & Norm & Skew- $t$ \\
\hline $\mathrm{AR}(3) \mathrm{TCM}$ & 0.182 & 0.190 & 0.210 & 0.202 & 0.154 & 0.160 & 0.168 & 0.179 \\
\hline AR(3) Mixt-TCM & 0.179 & 0.189 & 0.209 & 0.200 & 0.153 & 0.159 & 0.167 & 0.179 \\
\hline AR(3) DP-TCM & 0.178 & 0.188 & 0.210 & 0.201 & 0.153 & 0.160 & 0.168 & 0.178 \\
\hline AR(3)-GARCH(1,1) CTM & 0.194 & 0.193 & 0.207 & 0.201 & 0.161 & 0.161 & 0.178 & 0.181 \\
\hline AR(3)-GARCH(1,1) Mix-TCM & 0.193 & 0.192 & 0.206 & 0.200 & 0.160 & 0.161 & 0.177 & 0.180 \\
\hline AR(3)-GARCH(1,1) DP-TCM & 0.193 & 0.190 & 0.205 & 0.198 & 0.159 & 0.160 & 0.176 & 0.180 \\
\hline
\end{tabular}
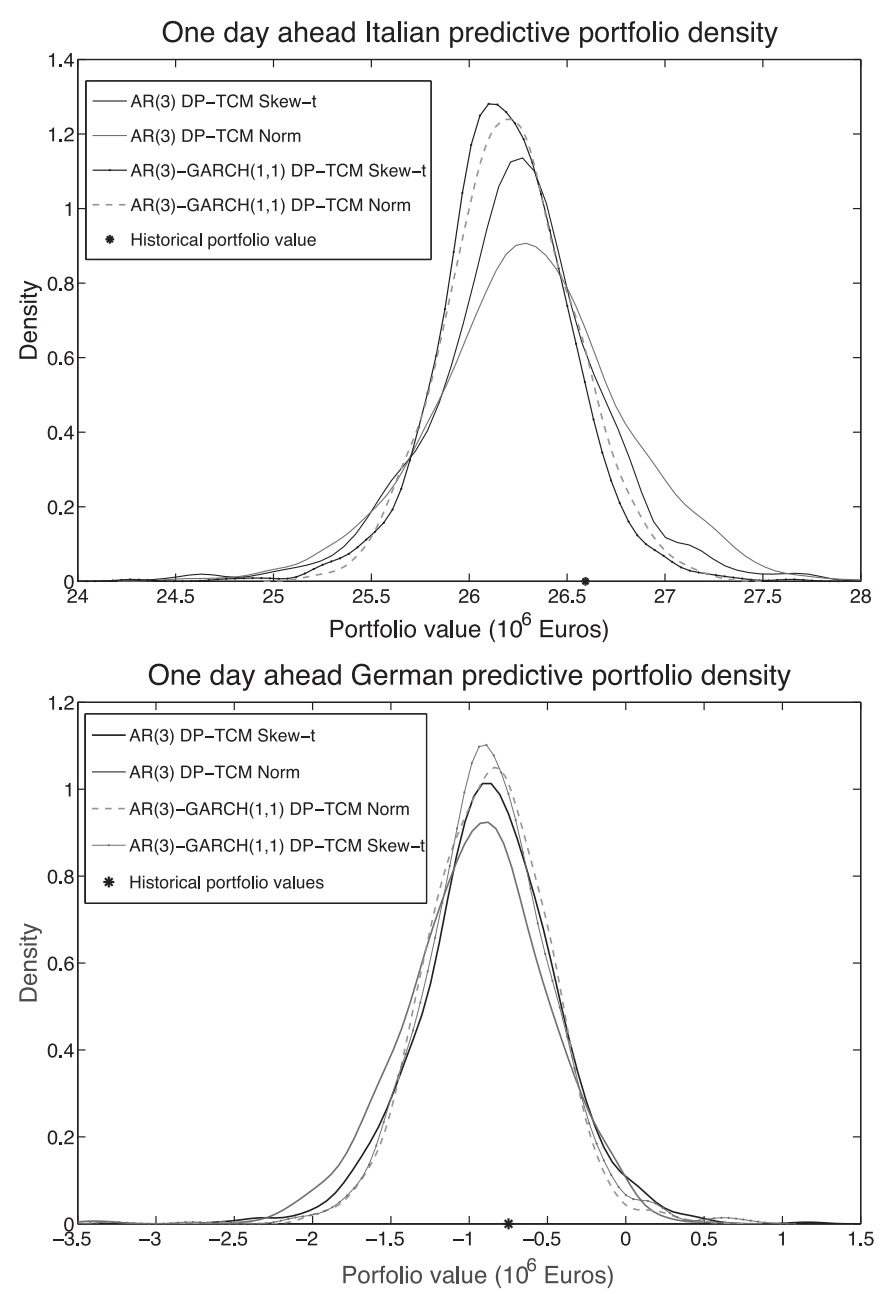

Fig. 10. One day ahead predictive distributions of the Italian and German portfolio values (15th December 2014).

For completeness, we tested our methodology using also alternative portfolio weights. Some results for an equally weighted portfolio are discussed in the last part of this subsection.

As described in Section 3.5, one can simulate the portfolio predictive distribution at time $T+1$ on the basis of the information up to time $T$ and, then, compute the related Bayesian predictive $V a R$ and $E S$. As an example, the predictive portfolio value distribution at time $T+1(T=15$ th December 2014) for Italian/German market for AR(3)-GARCH(1,1) and AR(3) DP-TCM with Skew-t and Normal margins are shown in Fig. 10. One can clearly note that the portfolio value corresponding to the AR(3) DP-TCM has larger variance with respect to the analogous portfolio estimated with $\mathrm{AR}(3)$ GARCH(1,1) DP-TCM. In all the cases the portfolio distribution cor-
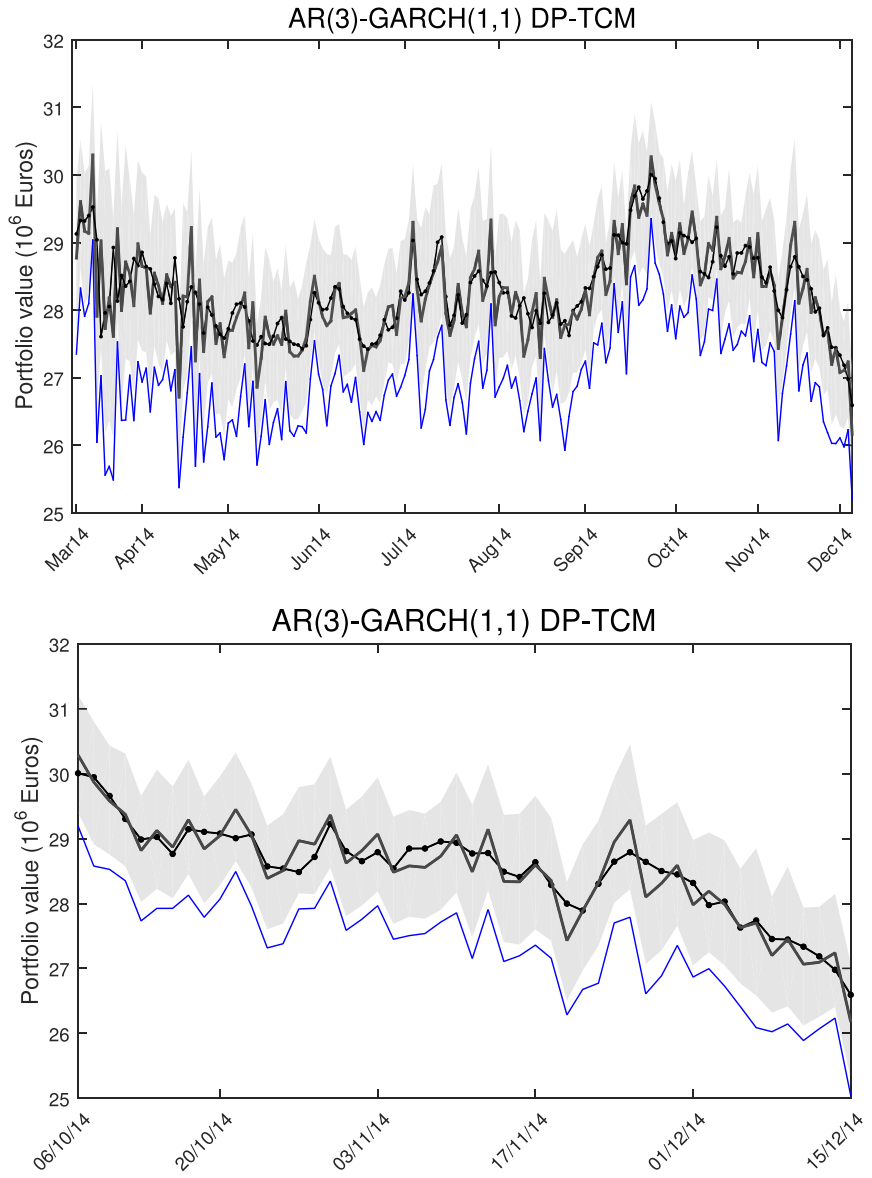

Fig. 11. One day ahead estimated Italian portfolio values (continuous black line) compared with the true portfolio values (dotted black line), with $99 \%$ credible intervals (grey areas) and minus the $99 \%$ ES (blue line) under the $\operatorname{AR}(3)-G A R C H(1,1)$ DP-TCM with Skew- $t$ margins. In sample (on the last 200 observations) first row, out of sample (on the last 50 observations) second row. (For interpretation of the references to colour in this figure legend, the reader is referred to the web version of this article.)

responding to models with Skew-t margins are more peaked although they show heavier tails.

We analysed the forecasting performance of our models using both in sample and an out of sample analysis for one day ahead portfolio value estimation. For the out of sample portfolio we considered the last 50 observations and we estimated the portfolio by the output of the MCMC algorithm (10,000 iterations) for each $t=174, \ldots, 224$. The estimated portfolios values are obtained by taking the predictive mean of the portfolio value, i.e. $\hat{V}_{t+1}=\mathbb{E}\left[V_{t+1} \mid \mathcal{O}_{t}\right]$. This value can be easily approximated by using the output of the MCMC. The results for the Italian and for the German portfolio obtained with the AR(3)-GARCH(1,1) DP-TCM models with Skew-t margins are reported in Figs. 11 and 12, 
Table 6

MAEs between the out of sample (on the last 50 observations) forecasting portfolio for the fully Bayesian $\mathrm{AR}(3)$ tree copula models and the historical portfolio.

\begin{tabular}{|c|c|c|c|c|c|c|c|c|}
\hline & \multicolumn{4}{|c|}{ "Realistic" portfolio } & \multicolumn{4}{|c|}{ Equally weighted portfolio } \\
\hline & \multicolumn{2}{|l|}{ Italy } & \multicolumn{2}{|c|}{ Germany } & \multicolumn{2}{|l|}{ Italy } & \multicolumn{2}{|c|}{ Germany } \\
\hline & Norm & Skew- $t$ & Norm & Skew- $t$ & Norm & Skew- $t$ & Norm & Skew- $t$ \\
\hline $\mathrm{AR}(3) \mathrm{TCM}$ & 0.179 & 0.181 & 0.208 & 0.200 & 0.155 & 0.159 & 0.171 & 0.178 \\
\hline AR(3) Mixt-TCM & 0.178 & 0.178 & 0.208 & 0.198 & 0.155 & 0.159 & 0.170 & 0.177 \\
\hline AR(3) DP-TCM & 0.174 & 0.176 & 0.205 & 0.195 & 0.153 & 0.158 & 0.169 & 0.176 \\
\hline
\end{tabular}
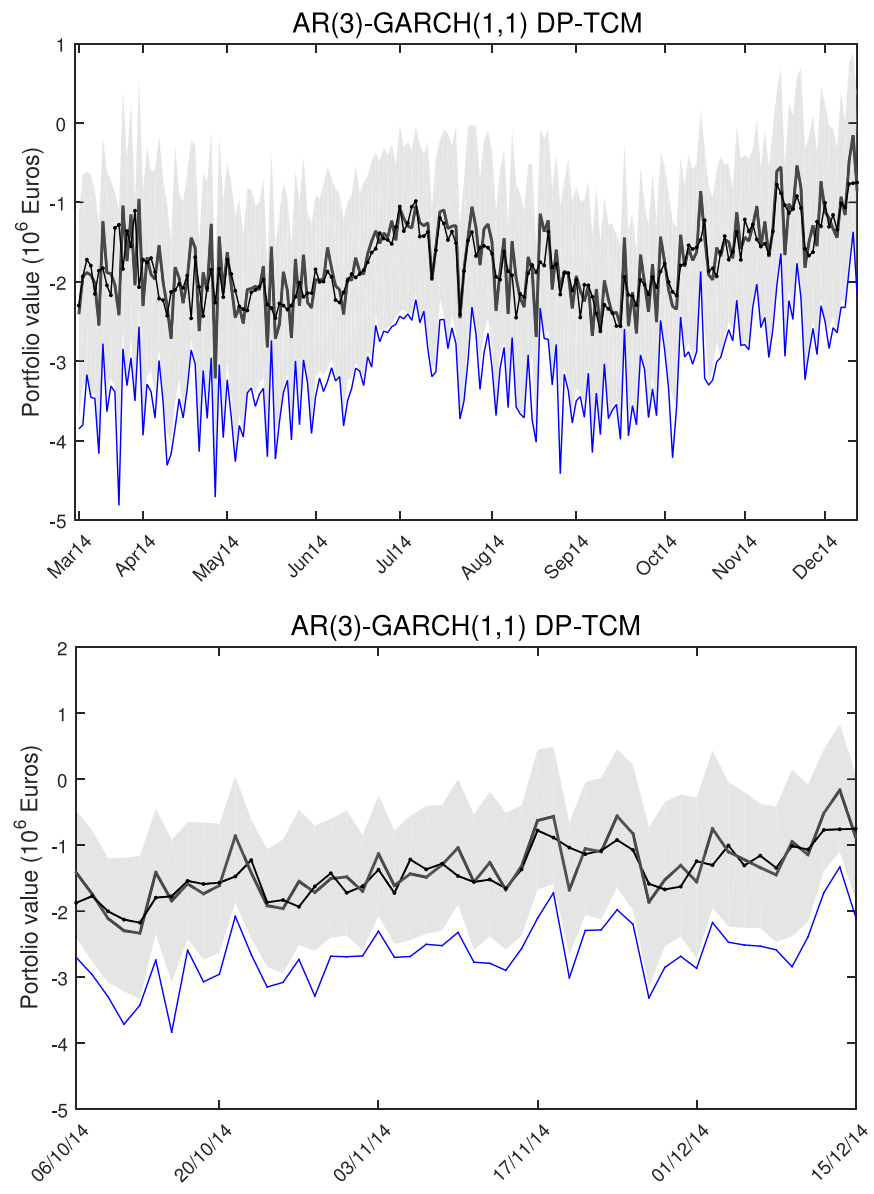

Fig. 12. One day ahead estimated German portfolio values (continuous black line) compared with the true portfolio values (dotted black line) with $99 \%$ credible intervals (grey areas) and minus the 99\% ES (blue line) under the AR(3)-GARCH(1,1) DP-TCM with Skew- $t$ margins. In sample (on the last 200 observations) first row, out of sample (on the last 50 observations) second row. (For interpretation of the references to colour in this figure legend, the reader is referred to the web version of this article.)

respectively. The estimated portfolio values (continuous line) are compared with the true portfolio values (dotted line). The $99 \%$ credible intervals of the estimated portfolio values are in grey and minus the $99 \%$ ES is plotted with a blue line. Clearly, minus the $99 \%$ VaR coincides with the lower bound of the grey areas. Overall, there is good agreement between the behaviours of predictive portfolios and the historical ones. In the out of sample case, the true values are always above minus the estimated 99\% VaR.

Finally, Table 5 reports the Mean Absolute Error (MAE) between the out of sample forecasting portfolio and the historical portfolio, that is

$\frac{1}{\tau_{1}-\tau_{0}} \sum_{t=\tau_{0}}^{\tau_{1}}\left|\mathbb{E}\left[V_{t+1} \mid \mathcal{O}_{t}\right]-V_{t+1}\right|$, where $\tau_{0}=174$ and $\tau_{1}=224$, corresponding to October 6, 2014 and December 15, 2014.

For the "realistic" portfolio the best performance in term of MAE is obtained by the AR(3)-DP-TCM with Normal margins for the Italian portfolio and by AR(3)-GARCH(1,1)-DP-TCM with Skew- $t$ margins for the German portfolio. It should be noted that the MAEs of the AR(3) models differ significantly from the corresponding MAEs for the AR(3)-GARCH(1,1) models in the Italian case, while in the German portfolio exercise all the MAEs are very close. In sample MAEs on 200 observations (see Table S13 in the Supplementary Material) show similar trends, in particular all the methods have similar MAEs when applied to the forecasting of the German portfolio. For this reason we computed the MAEs for other type of portfolio weights. As an example, in Table 5, we show the (out of sample) estimates for an equally weighted portfolio (see Table S13 in Supplementary Materials for the in sample results). For this portfolio, the trends are similar for both the Italian and the German market and the AR models show the best results in term of MAE (both in the in sample and in the out of sample experiments).

We conclude this subsection with a comparison of the previous results with the ones obtained with a fully Bayesian approach with AR tree copula models. In the fully Bayesian case, in addition to the priors (4)-(6) we also consider a Normal prior on the AR parameters:

$\boldsymbol{\alpha}_{k} \sim \mathcal{N}_{p}\left(\boldsymbol{M}_{\boldsymbol{k}}, \boldsymbol{\Sigma}_{\mathbf{k}}\right)$

where $\mathcal{N}_{p}(\boldsymbol{M}, \boldsymbol{\Sigma})$ is a $p$-dimensional Normal distribution with mean $\boldsymbol{M}$ and covariance matrix $\boldsymbol{\Sigma}$. Table 6 reports the MAEs between the out of sample forecasting portfolio values and the historical ones. It is worth noticing that the values are very similar to the ones obtained with the B-IFM procedures (see Table 5), although for the "realistic" portfolio they are slightly better. Overall, the improvement in the MAEs does not justify the additional computational work needed for a fully Bayesian estimation of the proposed models.

\section{Summary and concluding remarks}

The aim of this paper was to propose a novel Bayesian methodology for multivariate dependence analysis in the energy market. Our final goal was to study the connections between the main factors affecting energy price, and to provide efficient tools for portfolio risk evaluations.

We presented a Bayesian analysis of AR-GARCH copula models, in which the joint distribution of the standardised residuals of a panel of AR-GARCH time series is described via suitable tree copula models. Tree copulas are a particular type of R-vines, whose simple underlying graphical structure allows for an efficient inferential engine. In addition, via the graphical representation of the model, dependencies among the variables can be easily explained to nonexperts. Nevertheless, in some cases the independence constraints implied by the tree structure can be too stringent. For this reason, we also considered mixtures of tree copulas. Using this strategy we preserved the relative low complexity of the tree copula structures, taking into account richer dependencies between the variables. We examined both the case in which the joint distribution 
of the standardised residuals is represented via a finite mixture of tree copulas and by an infinite mixture.

We applied our methodology to the analysis of two representative European markets, the Italian and German one. Our data are daily observations of one-year forward contracts. Due to the nature of the data, the dependence structure among variables can differ significantly from one year to another. Hence, we focused on a single year at the time. We presented a detailed analysis of year 2014 for both markets, and we provided a multi-year analysis of the Italian market from 2013 to 2016. We select this specific time period to to investigate the effect of the entrance of Italy in the EEX market in 2014. The estimated dependence structure are in line with specific characteristics of the current energy market. Bayesian predictive estimates of standard risk measures, i.e. VaR and $E S$, together with portfolio predictive distribution are easily obtained from the MCMC output.

Even if in our models we assumed that the marginal distributions of the standardised residuals are Normal or Skew- $t$ and the linking copulas are Double Clayton and/or Double Gumbel, the methodology and the corresponding computational algorithms can be easily adapted to employ other types of copulas and/or marginal distributions.

\section{Acknowledgements}

The authors would like to thank the Referees and the Associate Editor for their very careful reading of our manuscript and the manifold pieces of advice for improvement. The authors are grateful to ENEL Group for providing the data, and for helpful comments and discussions. We also would like to thank Erika Blanc and Giorgia Oggioni for helpful comments and suggestions. The work was financially supported by the project "Multivariate Statistical Analysis for Extreme Value Risk Management in Energy Markets" (Contratto Aperto 8400057081) by Enel S.p.A. The work of the first author was partially supported by MIUR, Italy, PRIN 2015SNS29B-002. The work of the second author was partially supported by MIUR, Italy, PRIN MISURA 2010RHAHPL.

\section{Supplementary material}

Supplementary material associated with this article can be found, in the online version, at doi: 10.1016/j.ejor.2018.02.037.

\section{References}

Aas, K., Czado, C., Frigessi, A., \& Bakken, H. (2009). Pair-copula constructions of multiple dependence. Insurance: Mathematics and Economics, 44, 182-198.

Acerbi, C., \& Tasche, D. (2002). Expected shortfall: A natural coherent alternative to value-at-risk. Economic Notes, 31, 379-388.

Arreola Hernandez, J. (2014). Are oil and gas stocks from the australian market riskier than coal and uranium stocks? Dependence risk analysis and portfolio optimization. Energy Economics, 45, 528-536.

Artzner, P., Delbaen, F., Eber, J. M., \& Heath, D. (1999). Coherent measures of risk. Mathematical Finance, 9, 203-228.

Basel Committee on Banking Supervision (1995). An internal model-based approachto market risk capital requirements. In Bank for international settlements. Basel, Switzerland.

Bedford, T., \& Cooke, R. M. (2001). Probability density decomposition for conditionally dependent random variables modeled by vines. Annals of Mathematics and Artificial Intelligence, 32, 245-268.

Bedford, T., \& Cooke, R. M. (2002). Vines - a new graphical model for

dependent random variables. The Annals of Statistics, 30, 1031-1068.

Celeux, G., Forbes, F., Robert, C. P. \& Titterington, D. M. (2006). Deviance information criteria for missing data models. Bayesian Analysis, 1, 651-673.
Czado, C., Gärtner, F., \& Min, A. (2011). Analysis of australian electricity loads using joint Bayesian inference of d-vines with autoregressive margins. In Dependence modeling: Vine copula handbook (pp. 265-280). London: World

Scientific.
Czado, C., \& Min, A. (2011). Bayesian model selction fo d-vine pair copula constructions. The Canadian Journal of Statistics, 39, 239-258.

Dalla Valle, L., De Giuli, M. E., Tarantola, C., \& Manelli, C. (2016). Default probability estimation via pair copula constructions. European Journal of Operational Research, 249, 298-311.

Demarta, S., \& McNeil, A. (2005). The t copula and related copulas. International Statistical Review, 73(1), 111-129.

Elidan, G. (2013). Copulas and machine learning. In Copulae in mathematical and quantitative finance (pp. 39-60). Springer Berlin.

Embrechts, P., Puccetti, G., Rüschendorf, L., Wang, R., \& Beleraj, A. (2014). An academic response to basel 3.5. Risks, 2, 25-48.

Emmer, S., Kratz, M., \& Tasche, D. (2015). What is the best risk measure in practice? A comparison of standard measures. Journal of Risk, 18, 31-60.

Ferguson, T. S. (1973). A Bayesian analysis of some nonparametric problems. The Annals of Statistics, 1(2), 209-230.

Geweke, J. (1993). Bayesian treatment of the independent student-t linear model. Journal of Applied Econometrics, 8, S19-S40.

Gneiting, T., \& Raftery, A. E. (2007). Strictly proper scoring rules, prediction, and estimation. Journal of the American Statistical Association, 102, 359-378. Gruber, L. F., \& Czado, C. (2014). Bayesian model selection of regular vine copulas. In The Contribution of Young Researchers to Bayesian Statistics: 63 (pp. 177-180).

Gruber, L. F., \& Czado, C. (2015). Sequential Bayesian model selection of regular vine copulas. In Bayesian analysis: 10 (pp. 937-963). Hansen, B. E. (1994). Autoregressive conditional density estimation. International Economic Review, 35(3), 705-730.

Hofmann, M., \& Czado, C. (2010). Assessing the var of a portfolio using d-vine copula based multivariate GARCH models. Technische Universitaät München Zentrum Mathematik Working paper.

Ishwaran, H., \& Zarepour, M. (2002). Exact and approximate sum representations for the Dirichlet process. Canadian Journal of Statistics, 30, 269-283.

Jaschke, S. (2014). Estimation of risk measures in energy portfolios using modern copula techniques. Computational Statistics \& Data Analysis, 76, 359-376.

Joe, H. (1996). Families of $m$-variate distributions with given margins and $m(m-1) / 2$ bivariate dependence parameters. IMS Lecture Notes-Monograph Series, 28 , 120-141.

Joe, H. (1997). Multivariate model and dependence concepts. In Monographs on statistics an applied probability: Vol. 73. Chapman, Hall, London.

Joe, H. (2005). Asymptotic efficiency of the two-stage estimation method for copula-based models. Journal of Multivariate Analysis, 94, 401-419.

Kalli, M., Griffin, J. E., \& Walker, S. G. (2011). Slice sampling mixture models. Statistics and Computing, 21(1), 93-105.

Kirshner, S. (2007). Learning with tree-averaged densities and distribution. In Advances in neural information processing systems: Vol. 20.

Klugman, S. A., Panjer, H. H., \& Willmot, G. E. (2008). Loss models: From data

to decisions. NJ: John Wiley \& Sons.

Kurowicka, D. (2011). Optimal truncation of vines. In Dependence modeling: Vine copula handbook. World Scientific Publishing Co.

Laih, Y. W. (2014). Measuring rank correlation coefficients between financial time series: A GARCH-copula based sequence alignment algorithm. European Journal of Operational Research, 232, 375-382.

Lauritzen, S. (1996). Graphical models. Oxford: Clarendon Press.

Marimoutou, V., \& Soury, M. (2015). Energy markets and $\mathrm{CO}_{2}$ emissions: Analysis

by stochastic copula autoregressive model. Energy, 88, 417-429.

Meil ă, M., \& Jaakkola, T. (2006). Tractable bayesian learning of tree belief networks. In Statistics and computing: Vol. 16 (pp. 77-92).

Meil ă, M., \& Jordan, M. (20 00 0). Learning with mixtures of trees. Journal of Machine Learning Research, 1, 1-48.

Min, A., \& Czado, C. (2010). Bayesian inference for multivariate copulas using pair-copula constructions. Journal of Financial Econometrics, 8, 511-546.

Oh, D. H., \& Patton, A. J. (2017). Modelling dependence in high dimensions with factor copulas. Journal of Business \& Economic Statistic, 35, 139-145.

Osiewalski, J., \& Pajor, A. (2010). Bayesian value-at-risk for a portfolio: Multiand univariate approaches using MSF-SBEKK models. Central European Journal of Economic Modelling and Econometrics, 2, 253-277.

Reinaud, J. (2007). $\mathrm{CO}_{2}$ allowance \& electricity price interaction. In Impact on industry's electricity purchasing strategies in Europe. IEA information paper

Richardson, S., et al. (2002). Discussion of spiegelhalter. Journal of the Royal Statistical Society, Series B, 64, 651-655.

Robert, C., \& Casella, G. (2004). Monte Carlo statistical methods. Springer.

Sethuraman, J. (1994). A constructive definition of Dirichlet priors. Statistica Sinica, 639-650.

Silva, R., \& Gramcy, R. B. (2009). MCMC methods for Bayesian mixtures of copula. Journal of Machine Learning Research, 5, 512-519.

Sklar, A. (1959). Fonctions de répartition á ndimensions et leurs marges. In Publications de l'institut de statistique de l'université de paris: Vol. 8 (pp. 229-231).

Spiegelhalter, D. J., Best, N. G., Carlin, B. P., \& van der Linde, A. (2002).

Bayesian measures of model complexity and fit (with discussion). Journal of the Royal Statistical Society, Series B, 64, 583-639.

Szegö, G. (2005). Measures of risk. European Journal of Operational Research, 163, 5-19.

Van Dyk, D. A., \& Meng, X. L. (2001). The art of data augmentation. Journal of Computational and Graphical Statistics, 10, 1-50. 
Walker, S. G. (2007). Sampling the Dirichlet mixture model with slices. Communications in Statistic - Simulation and Computation, 36(1), 45-54.

Wang, G. J., \& Xie, C. (2016). Tail dependence structure of the foreign exchange market: A network view. Expert Systems with Applications, 46, 164-179.
Wen, X., Wei, Y., \& Huang, D. (2012). Measuring contagion between energy market and stock market during financial crisis: A copula approach. Energy Economics, $34, \quad 1435-1446$.

Wu, C. C., Chung, H., \& Chang, Y. H. (2012). The economic value of co-

movement between oil price and exchange rate using copula-based GARCH models. Energy Economics, 34, 270-282. 